Mon. Not. R. Astron. Soc. 000,114(2013) Printed 9 September $2013 \quad$ (MN LATEX style file v2.2)

\title{
Unveiling new members in five nearby young moving groups
}

\author{
A. Moór ${ }^{1 \star}$, Gy. M. Szabó ${ }^{1,2,3}$, L. L. Kiss ${ }^{1,2,4}$, Cs. Kiss $^{1}$, P. Ábrahám ${ }^{1}$, J. Szulágyi ${ }^{1}$
}

Á. Kóspál ${ }^{5}$, T. Szalai ${ }^{3}$

${ }^{1}$ Konkoly Observatory, Research Centre for Astronomy and Earth Sciences,

Hungarian Academy of Sciences, PO Box 67, H-1525 Budapest, Hungary

${ }^{2}$ ELTE Gothard-Lendület Research Group, 9700 Szombathely, Hungary

${ }^{3}$ Dept. of Experimental Physics and Astronomical Observatory, 6720 Szeged Dóm tér 9., Hungary

${ }^{4}$ Sydney Institute for Astronomy, School of Physics, University of Sydney, NSW 2006, Australia

${ }^{5}$ Research and Scientific Support Department, European Space Agency

(ESA-ESTEC, SRE-SA),

P.O. Box 299, 2200 AG, Noordwijk, The Netherlands ; ESA fellow.

Accepted ... Received ...; in original form ...

\begin{abstract}
In the last decade many kinematic groups of young stars $(<100 \mathrm{Myr})$ were discovered in the solar neighbourhood. Since the most interesting period of planet formation overlaps with the age of these groups, their well dated members are attractive targets for exoplanet searches by direct imaging. We combined astrometric, photometric and X-ray data, and applied strict selection criteria to explore the stellar content of five nearby moving groups. We identified more than 100 potential new candidate members in the $\beta$ Pic moving group, and in the Tucana-Horologium, Columba, Carina, and Argus associations. In order to further assess and confirm their membership status, we analysed radial velocity data and lithium equivalent widths extracted from highresolution spectra of 54 candidate stars. We identified 35 new probable/possible young moving group members: 4 in the $\beta$ Pic moving group, 11 in the Columba association, 16 in the Carina association, and 4 in the Argus association. We found serendipitiously a new AB Dor moving group member as well. For four Columba systems Hipparcos based parallaxes have already been available and as they are consistent with the predicted kinematic parallaxes, they can be considered as secure new members.
\end{abstract}

Key words: stars: kinematics - open clusters and associations: individual: $\beta$ Pictoris moving group, Tucana-Horologium association, Columba association, Carina association, Argus association.

\section{INTRODUCTION}

Nearby young moving groups are gravitationally unbound, loose associations of stars that have common origin and move through space together. These groups occupy extended regions $(\sim 100 \mathrm{pc})$ in our Solar System's neighbourhood and due to their proximity the group members appear to us widely spread across the sky. This makes the integrity of the systems hardly recognizable. Members of a specific group can be linked together through their common space velocities and common ages. Such a study requires the combination of astrometric data with radial velocity information and

* E-mail:moor@konkoly.hu application of relevant age diagnostic methods. This is why the intensive investigation of nearby young moving groups started only in the late nineties of the last century, after the Hipparcos and TYCHO all-sky astrometric catalogues and the ROSAT all-sky point source catalogues (X-ray activity can be used as a youth indicator) became available. Thanks to subsequent studies, up to now nine young $(<100 \mathrm{Myr})$ kinematic groups have been identified in the vicinity of the Sun (for a review, see Zuckerman \& Song 2004; Torres et al. 2008).

According to the review by Torres et al. (2008), more than 300 members of these nine groups are known. The census of these groups is far from complete, because the necessary kinematic information, particularly radial veloc- 
ities and trigonometric distances, are missing for most of the nearby stars. This is especially true for low-mass populations of moving groups, whose census may be highly incomplete (e.g. Shkolnik, Liu, \& Reid 2009). Thanks to studies focused on searching for such young low mass stars, a large number of new M-type group members/candidates were identified in the last few years (Malo et al. 2013; Schlieder, Lépine, \& Simon 2010, 2012a,b; Shkolnik et al. 2011, 2012). The census of the high mass regime could, however, be incomplete as well, as demonstrated by recent discoveries of several new early-type members of these groups (Zuckerman et al. 2011; Zuckerman \& Song 2012).

A significant fraction of nearby stars younger than 100 Myr belongs to the known kinematic groups. The ages of these nearby young moving groups range between $\sim 6$ and 100 million year, thus - according to the current paradigm - overlap with the most interesting period of planetary system formation. Gas giant planets must form before the gas content of the primordial protoplanetary disc is largely dissipated (i.e. within $10 \mathrm{Myr}$, Pascucci et al. 2006), while terrestrial planets may reach their final mass on time-scales of ten millions of years (Chambers 2001; Kenvon \& Bromlev 2006). Although the formation of planetesimals and planetesimal belts in the outer region of planetary systems may even last for hundred million years, the most active period is restricted to the first few tens of million years (Kenvon \& Bromlev 2008). The evolution of planetesimal belts can be traced via the investigation of debris disks that are linked to them (Wyatt 2008). Thus, members of nearby young moving groups are suitable targets to study the evolution of planetary systems, particularly because, unlike field stars, their ages are well determined. These young kinematic groups also offer insights into the star formation process in low-density environments (Fernández, Figueras \& Torra 2008).

Young stars are excellent targets to detect sub-stellar objects via direct imaging, since giant gas planets are thought to have significant excess luminosity due to the rapid contraction in the early phase of their evolution (Kasper et al. 2007). Indeed, HR 8799 - a star with a multiple planetary system discovered via direct imaging (Marois et al. 2008) - may belong to the 30 Myr old Columba association. $\kappa$ And, hosting star of a super-Jupiter successfully imaged with Subaru/HiCIAO, can also be assigned to the Columba association (Carson et al. 2013). $\beta$ Pic, the member and namesake of the $\beta$ Pic moving group, also harbours an imaged planet (Lagrange et al. 2010).

In our study, we searched for new members in five young assemblages, the $\beta$ Pic moving group (BPMG), the Tucana-Horologium (THA), the Columba (COL), the Carina (CAR), and the Argus (ARG) associations. Each selected kinematic group is younger than $50 \mathrm{Myr}$ and most of their known members are located within $120 \mathrm{pc}$ to the Sun. In Appendix A, we briefly summarise the main properties of the selected young associations. We selected potential candidate members from a catalogue compiled by crosscorrelating the entries of the Naval Observatory Merged Astrometric Dataset (NOMAD, Zacharias et al. 2004a) with the entries of the ROSAT All-Sky Bright Source Catalogue and the ROSAT All-Sky Survey Faint Source Cata$\log$ (Voges et al. 1999, 2000) (Sect. 2.1). Due to the selection method applied our survey was most efficient in finding
F5-M type group members. In order to confirm the membership of our potential candidates, we performed follow-up high-resolution spectroscopy (Sect. 3). The analysis of these spectra and the final assignments of the candidate stars are reviewed in Sect. 4 our results are summarized in Sect. 5 .

\section{CANDIDATE SELECTION}

\subsection{Selection method}

Young stars with spectral type $\mathrm{F}$ and later are known to exhibit enhanced coronal activity with strong X-ray emission, making the latter property a good indicator of youth (Zuckerman \& Song 2004). Exploiting this characteristic as a first step of our survey, we cross-correlated the entries of the NOMAD catalogue with the entries of ROSAT catalogues compiling an initial list of potential young stars from the whole sky. For real coincidences we demanded that the NOMAD counterpart(s) must be located within a distance of less than 2 times the ROSAT positional error from the X-ray source's position. We included only those stars whose proper motion measurement fulfilled the following criteria: 1) $\mu=\sqrt{\mu_{\alpha}^{2} \cos \delta^{2}+\mu_{\delta}^{2}}>20$ mas $\mathrm{yr}^{-1}$; 2) $\mu / \sigma_{\mu}>5$. Because of the selection method, the compiled sample contains primarily stars with spectral type F5 or later. In order to select the most probable candidate members of the chosen five young moving groups, this starting sample of likely young stars was further analysed.

Stars that belong to a specific kinematic group share common spatial motion through the Milky Way. Due to projection effects, the common space motion manifests itself as a converging pattern, and the motion of the members defines a common point of convergence in the sky. Thus, based on positional and proper motion data, one can isolate possible members of a group. We used the proper motion selection algorithm proposed by Lépine \& Simon (2009) to identify objects with proper motions consistent with membership in any of our five selected groups. Positional and proper motion data of candidate sources were based on the NOMAD catalogue, the characteristic galactic space motion of selected young moving groups was taken from Torres et al. (2008). For each of our candidates, we calculated the projected motion of all five groups in the plane of the sky local to the star and then computed the $\Phi$ angle that these vectors subtend with the candidate's proper motion vector (Eq. 1-3 in Lépine \& Simon 2009). The smaller the value of $\Phi$ the better the consistency between the motion of the candidate star and the characteristic motion of a kinematic group. In order to determine an upper threshold for $\Phi$, we repeated these calculations for the known members of the selected five groups (the memberlist was taken from Torres et al. 2008). We found that for more than $90 \%$ of known members the value of $\Phi$ is less than $12^{\circ}$. Adopting this value as an upper threshold we omitted all those stars from the starting sample whose proper motion was not consistent with the mean projected proper motion of any of the selected moving groups. Assuming that a certain star belongs to a specific kinematic group, we estimated a kinematic parallax for all of the remaining candidates and predicted their radial velocity as well (Eq. 6 and 8 in Lépine \& Simon 2009).

To refine the list of candidates further, we derived K- 
band absolute magnitudes from the kinematic distances $\left(M_{K s, k i n}\right)$ or from the trigonometric distances $\left(M_{K s, t r i}\right)$ when Hipparcos data were available. These were compared with the absolute photometric K magnitudes $\left(M_{K s, p h o}\right)$ calculated from observational isochrones. The observational isochrones were defined using the colour-magnitude diagram $\left(M_{K s}\right.$ vs. $\left.V-K_{s}\right)$ of the specific groups where the locus of the known members was fitted with a linear relationship (see e.g. Fig. 1). The members of the co-eval THA, COL, and CAR groups were fitted synchronously. For true moving group members, $M_{K s, k i n}$ (or $M_{K s, t r i}$ ) and $M_{K s, p h o}$ must be consistent. Based on the known members we found that $\left|\Delta M_{K s}\right|=\left|M_{K s, \text { kin } / \text { tri }}-M_{K s, p h o}\right| \leqslant 1 \mathrm{mag}$, therefore, candidate objects with $\left|\Delta M_{K s}\right|>1$ mag were discarded from the sample.

Using the kinematic distances, we computed the candidates' physical space coordinates centred on the Sun (X,Y,Z) and compared them to the region defined by the known members of the assigned group. In most cases the boundaries of a specific group's region (ranges in X, Y, Z coordinates) were taken from Torres et al. (2008, see their Table 2). In Moór et al. (2011) we reported the discovery of a new probable Columba member, HD 3670, which is located slightly out of the region defined in the abovementioned work. Taking into account the position of this new member for Columba, we adopted a region that is somewhat more extended in the $\mathrm{X}$ direction $(\mathrm{X}=-106-+20 \mathrm{pc})$ than that defined in Torres et al. (2008, $X=-106-+9 \mathrm{pc}$ ). In the following, we ignore candidates located outside the defined regions.

Our sample consists of stars that have X-ray counterparts. However, even quite old stars can exhibit X-ray emission and could be detected by ROSAT. Rotation and activity of sun-like main sequence stars diminish during their life. Potential young star can be selected based on their enhanced coronal activity that is observable in their strong Xray emission and high fractional X-ray luminosity $\left(L_{\mathrm{x}} / L_{\mathrm{bol}}\right)$. We plotted fractional X-ray luminosities of candidate stars as a function of their $V-K_{s}$ colour indices and compared them with the distribution of known members in the same plot. Since the X-ray activity of known members of the selected groups as a function of $V-K_{s}$ are very similar to each other, in this case we used them as an ensemble of young stars and did not separate them based on their group membership. After this comparison we kept only those candidates whose X-ray properties were consistent with that of known members (see e.g. Fig. 2).

As a final step of our selection process, we searched the literature for additional data on our candidate stars and rejected those targets that 1) have already been identified as a member of a young moving group; 2) are included in the Hipparcos catalogue and their measured trigonometric parallaxes are not consistent with the kinematic ones; 3) have radial velocity data inconsistent with the predicted ones; 4) reside in a binary system, where the companion has a measured trigonometric parallax and/or radial velocity which is/are inconsistent with the predicted value(s). As a result of the applied selection algorithm, we finally identified 129 stars that could potentially be assigned to at least one of the selected five young moving groups. There are several stars which could be assigned to more than one group.

Members of a young moving group are expected to share a similar space motion. Consistency between the proper mo- tion of a candidate and the projected motion of a moving group is a necessary but not sufficient condition of the membership, since e.g. stars can move in the same direction but with a significantly different speed. The computation of the heliocentric space motion $(\mathrm{U}, \mathrm{V}, \mathrm{W})$ of a star requires the knowledge of both its radial velocity and distance. X-ray activity of stars measured by their fractional X-ray luminosity is a useful youth indicator, however, it is not applicable as an accurate age diagnostic for $10-100$ Myr old stars since in this range this property shows a weak dependency on age. By measuring the radial velocity of the candidates and by applying additional age diagnostic methods (e.g. lithium content), we can collect further evidences for their membership.

We obtained high-resolution spectra for fifty candidate stars from the compiled list. For four additional objects we used data from the SACY survey (Torres et al. 2006) to complement our observed sample. Hereafter, throughout this paper, we focus on the analysis of these 54 stars.

\subsection{Basic properties of the selected stars}

Basic properties of the 54 stars as well as their suspected host kinematic groups are summarized in Table 1 There are several stars for which more than one kinematic groups are listed. We note that for these 54 targets, we also investigated the possibility whether they could be the member of three additional groups: the TW Hya association, the AB Doradus moving group, and the $\eta$ Cha group. In Table 1 for a certain candidate star, we list the relevant groups in ascending order of $\Phi$ values (smaller $\Phi$ indicates better consistency of the motion of the candidate star with the characteristic motion of a kinematic group).

In order to estimate the effective temperature of our targets, we modelled the stellar photosphere by fitting the optical and infrared photometric data with a NEXTGEN atmosphere model (Hauschildt, Allard, \& Baron 1999). Optical photometry, when available, were taken from the $T Y$ $\mathrm{CHO} 2$ catalogue, otherwise we used the $V$ magnitude quoted in the NOMAD catalogue. Additional near-infrared data in $J, H, K_{s}$ bands were taken from the Two Micron AllSky Survey (2MASS; Skrutskie et al. 2006). This data set was further supplemented by Wide-field Infrared Survey Explorer (WISE) $W 1$ band (centred at $3.4 \mu \mathrm{m}$ ) photometry from the WISE All-Sky Database (Wright et al. 2010). The surface gravity was fixed to $\log g=4.5 \mathrm{cms}^{-2}$ and we adopted solar metallicity for all of our targets. Since all of our stars are predicted to be located within $125 \mathrm{pc}$ (i.e. most of them can be found inside the Local Bubble, where the mean extinction is low, Lallement et al. 2003) the visual extinction was neglected in the fitting process. The $T_{\text {eff }}$ values obtained are listed in Table 1 .

\section{OBSERVATIONS AND DATA REDUCTION}

\subsection{Spectroscopic observations}

Our sample includes 54 candidates. For four of them, spectroscopic data were taken from the SACY catalogue (Torres et al. 2006). For the remaining 50 selected candidate 
Table 1. Basic properties of candidate members. (1) NOMAD-identifier of the star. (2) Other, SIMBAD compatible, name of the star. (3) Right ascension (J2000). (4) Declination (J2000). (5) Spectral type, taken from SIMBAD if available, otherwise (marked by asterisks) estimated from the derived effective temperature. For the latter calculation we used the conversion tables from Pecaut \& Mamaiek (2013): for stars that we identified as a probable member of young moving groups (see Sect.4.1.3) we used their table 4 (with $\mathrm{T}_{\text {eff-spectral type }}$ tabulation for 5-30 Myr pre-main-sequence stars), in other cases the $\mathrm{T}_{\text {eff }}$ and spectral type data were taken from their table 6 (tabulation for dwarfs). (6) 2MASS $\mathrm{K}_{s}$ magnitude. (7) V-K $\mathrm{K}_{s}$ colour index. (8) Derived effective temperature. (9) Fractional X-ray luminosity based on ROSAT data. 0466-0044732, 0466-0044706 and 0601-0968580 are members of binary systems where both components are within the positional uncertainty circle of a nearby ROSAT source. To account for binarity, in these cases the X-ray flux was divided by two. (10) Identifiers of candidate moving groups. There are several stars that could be assigned to more than one group. In these cases the groups are listed in increasing order of $\Phi$ values.

\begin{tabular}{|c|c|c|c|c|c|c|c|c|c|}
\hline $\begin{array}{c}\text { NOMAD ID } \\
\text { (1) }\end{array}$ & Other name & $\begin{array}{c}\text { RA (2000) } \\
(3)\end{array}$ & $\begin{array}{c}\text { DEC (2000) } \\
(4)\end{array}$ & $\begin{array}{l}\mathrm{SpT} \\
(5)\end{array}$ & $\begin{array}{c}\mathrm{K}_{s} \\
{[\mathrm{mag}]} \\
(6)\end{array}$ & $\begin{array}{c}\mathrm{V}-\mathrm{K}_{s} \\
{[\mathrm{mag}]} \\
(7)\end{array}$ & $\begin{array}{c}T_{e f f} \\
{[\mathrm{~K}]} \\
(8)\end{array}$ & $\begin{array}{c}\log \frac{L_{\mathrm{X}}}{L_{\mathrm{bol}}} \\
(9)\end{array}$ & Candidate groups \\
\hline 0367-0004557 & HD 3888 & $00: 40: 51.6$ & $-53: 12: 35.7$ & F7V & 6.11 & 1.26 & 6250 & -4.47 & $\mathrm{COL} / \mathrm{THA} / \mathrm{ABD}$ \\
\hline 0361-0008966 & HD 8077 & 01:19:05.6 & $-53: 51: 01.9$ & F6V & 7.61 & 1.27 & 6200 & -4.05 & $\mathrm{COL}$ \\
\hline $0540-0027893$ & CD-36 1289 & $03: 25: 51.9$ & $-35: 56: 25.5$ & $\mathrm{~F} 8 \mathrm{~V}$ & 8.14 & 1.74 & 5400 & -3.23 & $\mathrm{COL}$ \\
\hline 0453-0032480 & CD-45 1384 & 04:03:53.4 & $-44: 39: 31.6$ & G5* & 8.51 & 1.71 & 5450 & -2.98 & $\mathrm{COL}$ \\
\hline 0454-0034540 & CD-44 1533 & $04: 22: 45.7$ & $-44: 32: 51.9$ & $\mathrm{KOV}$ & 8.58 & 1.87 & 5400 & -3.36 & $\mathrm{COL}$ \\
\hline 0466-0044706 & HIP 25434 & $05: 26: 23.0$ & $-43: 22: 36.3$ & G0 & 7.76 & 1.29 & 6350 & -4.07 & $\mathrm{COL}$ \\
\hline 0466-0044732 & HIP 25436 & $05: 26: 24.0$ & $-43: 22: 32.7$ & $\mathrm{~F} 4 \mathrm{IV} / \mathrm{V}$ & 7.21 & 1.03 & 6700 & -4.35 & $\mathrm{COL}$ \\
\hline 0211-0114754 & HD 269620 & $05: 29: 27.1$ & $-68: 52: 04.9$ & G6V & 8.06 & 1.55 & 5700 & -3.72 & $\mathrm{COL}$ \\
\hline 0298-0039269 & HD 37402 & $05: 34: 26.1$ & $-60: 06: 15.1$ & $\mathrm{~F} 6 \mathrm{~V}$ & 7.09 & 1.29 & 6200 & -4.36 & $\mathrm{COL}$ \\
\hline 0211-0129478 & HD 269921 & $05: 38: 34.5$ & $-68: 53: 06.6$ & G7V & 8.39 & 1.81 & 5400 & -3.21 & COL \\
\hline 0404-0048765 & HD 38470 & $05: 43: 24.6$ & $-49: 31: 06.4$ & $\mathrm{~F} 4 \mathrm{~V}$ & 8.03 & 1.19 & 6400 & -4.37 & ARG \\
\hline 0351-0047432 & HD 39130 & $05: 47: 18.0$ & $-54: 50: 28.2$ & F6V & 7.43 & 1.26 & 6250 & -4.58 & $\mathrm{COL}$ \\
\hline 0287-0048860 & HD 44345 & $06: 17: 20.9$ & $-61: 14: 46.7$ & F5V & 7.39 & 1.11 & 6450 & -4.56 & CAR \\
\hline 0339-0066782 & TYC 8554-1187-1 & $07: 11: 57.3$ & $-56: 04: 21.5$ & $\mathrm{~F} 7^{*}$ & 8.97 & 1.22 & 6250 & -3.88 & $\mathrm{COL}$ \\
\hline 0315-0057897 & CD-58 1720 & $07: 20: 27.9$ & $-58: 29: 26.6$ & G3* & 8.44 & 1.63 & 5700 & -4.02 & CAR \\
\hline 0352-0070710 & CPD-54 1295 & $07: 31: 19.9$ & $-54: 47: 02.8$ & G5 & 8.75 & 1.75 & 5500 & -3.89 & $\mathrm{ARG}$ \\
\hline 0368-0077090 & - & $07: 39: 12.2$ & $-53: 07: 29.8$ & $\mathrm{~K} 6^{*}$ & 8.75 & 3.31 & 4200 & -3.30 & $\mathrm{COL}$ \\
\hline 0263-0084896 & CD-63 336 & $07: 42: 38.9$ & $-63: 36: 14.0$ & $\mathrm{G} 2^{*}$ & 8.39 & 1.53 & 5850 & -4.08 & $\mathrm{CAR} / \mathrm{COL}$ \\
\hline 0664-0176864 & CD-23 6751 & $08: 04: 24.8$ & $-23: 35: 00.2$ & K0 & 8.16 & 1.70 & 5500 & -3.63 & COL \\
\hline 0331-0114466 & - & $08: 09: 42.7$ & $-56: 52: 20.1$ & $\mathrm{KO}^{*}$ & 8.83 & 2.08 & 5050 & -3.30 & CAR \\
\hline 0406-0105889 & TYC 8157-91-1 & $08: 15: 21.6$ & $-49: 18: 30.3$ & $\mathrm{G} 7^{*}$ & 9.11 & 1.94 & 5300 & -3.60 & $\mathrm{COL}$ \\
\hline 0248-0110675 & TYC 8933-1204-1 & $08: 20: 56.0$ & $-65: 10: 12.4$ & $\mathrm{~K} 2 *$ & 8.93 & 2.49 & 4750 & -3.43 & $\mathrm{CAR} / \mathrm{COL}$ \\
\hline 0248-0115043 & HD 73129 & $08: 32: 46.2$ & $-65: 08: 27.2$ & $\mathrm{G} 2 \mathrm{~V}$ & 7.85 & 1.46 & 5850 & -4.35 & $\mathrm{ARG} / \mathrm{COL}$ \\
\hline 0354-0106699 & TYC 8573-579-1 & 08:41:01.3 & $-54: 31: 02.3$ & $\mathrm{G} 1^{*}$ & 9.01 & 1.39 & 5950 & -4.01 & $\mathrm{ARG}$ \\
\hline 0341-0108561 & - & $08: 44: 25.2$ & $-55: 48: 47.8$ & $\mathrm{~K} 3^{*}$ & 9.56 & 2.90 & 4450 & -3.29 & ARG \\
\hline 0489-0152365 & CD-40 4610 & $08: 46: 46.9$ & $-41: 05: 46.7$ & $\mathrm{~F} 8^{*}$ & 8.69 & 1.30 & 6100 & -4.00 & $\mathrm{COL}$ \\
\hline 0269-0122275 & - & $08: 52: 43.6$ & $-63: 04: 18.0$ & G3* & 8.68 & 1.65 & 5700 & -3.42 & $\mathrm{CAR}$ \\
\hline 0291-0099052 & TYC 8927-2869-1 & $08: 59: 04.5$ & $-60: 51: 46.6$ & G9* & 9.19 & 2.19 & 5100 & -3.52 & CAR \\
\hline $0372-0169783$ & - & 09:00:59.3 & $-52: 44: 45.4$ & $\mathrm{~K} 4^{*}$ & 9.42 & 2.96 & 4400 & -3.20 & $\mathrm{COL} / \mathrm{CAR}$ \\
\hline 0313-0102916 & TYC 8594-1049-1 & 09:01:45.6 & $-58: 41: 06.2$ & $\mathrm{~K} 0^{*}$ & 9.10 & 2.09 & 5000 & -3.27 & $\mathrm{COL} / \mathrm{CAR}$ \\
\hline 0397-0143387 & TYC 8174-1586-1 & $09: 11: 15.8$ & $-50: 14: 14.9$ & K5e & 9.50 & 2.46 & 4650 & -3.18 & $\mathrm{CAR} / \mathrm{COL}$ \\
\hline 0268-0125760 & TYC 8944-874-1 & $09: 11: 25.3$ & $-63: 11: 28.2$ & $\mathrm{~K} 2 *$ & 8.55 & 2.03 & 5050 & -3.27 & $\mathrm{COL} / \mathrm{ARG} / \mathrm{CAR}$ \\
\hline 0348-0138354 & TYC $8587-1015-1$ & $09: 15: 14.9$ & $-55: 11: 51.6$ & $\mathrm{KO}^{*}$ & 9.13 & 1.96 & 5300 & -3.30 & $\mathrm{CAR} / \mathrm{COL}$ \\
\hline 0308-0116109 & TYC 8595-1740-1 & $09: 15: 57.6$ & $-59: 11: 07.1$ & G5* & 9.22 & 1.83 & 5450 & -3.25 & $\mathrm{CAR} / \mathrm{COL}$ \\
\hline 0337-0139009 & TYC 8591-145-1 & $09: 17: 00.5$ & $-56: 16: 48.1$ & G9* & 9.08 & 1.94 & 5400 & -3.82 & $\mathrm{COL} / \mathrm{CAR}$ \\
\hline 0415-0155444 & HD 80595 & 09:19:04.2 & $-48: 24: 52.2$ & G0V & 8.17 & 1.40 & 6000 & -3.89 & $\mathrm{CAR} / \mathrm{COL}$ \\
\hline 0193-0211246 & TYC 9200-446-1 & 09:30:31.5 & $-70: 41: 48.0$ & G5* & 8.56 & 1.83 & 5500 & -3.51 & CAR/ARG \\
\hline 0367-0179130 & - & $09: 31: 25.4$ & $-53: 14: 36.6$ & $\mathrm{~K} 5^{*}$ & 9.50 & 3.31 & 4200 & -3.37 & $\mathrm{CAR} / \mathrm{COL}$ \\
\hline 0393-0168117 & - & $09: 36: 58.6$ & $-50: 41: 56.1$ & $\mathrm{~K} 2 *$ & 9.46 & 2.09 & 5000 & -3.16 & $\mathrm{COL} / \mathrm{CAR}$ \\
\hline 0327-0202270 & HD 302321 & $09: 41: 25.6$ & $-57: 15: 22.0$ & F8 & 8.42 & 1.43 & 5950 & -4.05 & $\mathrm{CAR} / \mathrm{COL}$ \\
\hline 0348-0190850 & HD 300458 & 10:04:57.4 & $-55: 10: 37.5$ & F8 & 8.27 & 1.62 & 5700 & -4.25 & ARG \\
\hline 0350-0195011 & TYC 8603-1896-1 & 10:06:11.0 & $-54: 58: 29.7$ & $\mathrm{~K} 2^{*}$ & 9.09 & 2.42 & 4950 & -3.59 & CAR \\
\hline 0154-0097276 & CD-73 584 & 10:30:03.5 & $-74: 32: 32.2$ & Ko & 8.19 & 1.91 & 5300 & -3.60 & $\mathrm{CAR} / \mathrm{ARG}$ \\
\hline 0349-0227761 & - & $10: 40: 10.6$ & $-55: 03: 42.4$ & $\mathrm{~K} 6^{*}$ & 9.45 & 3.40 & 4150 & -2.99 & ARG \\
\hline 0277-0339323 & TYC 8962-532-1 & $10: 56: 42.3$ & $-62: 17: 41.0$ & $\mathrm{~K} 6^{*}$ & 8.10 & 3.16 & 4250 & -3.66 & $\mathrm{COL} / \mathrm{CAR} / \mathrm{ARG}$ \\
\hline 0370-0341486 & TYC 8617-909-1 & $11: 23: 47.0$ & $-52: 57: 39.4$ & M0* & 7.95 & 3.71 & 3800 & -3.49 & $\mathrm{ARG} / \mathrm{COL}$ \\
\hline 0104-0056948 & TYC 9420-112-1 & $12: 36: 11.1$ & $-79: 31: 38.4$ & $\mathrm{~K} 3^{*}$ & 8.89 & 2.45 & 4650 & -3.73 & $\mathrm{ARG} / \mathrm{ABD}$ \\
\hline 0568-0654078 & - & $17: 06: 22.7$ & $-33: 08: 15.3$ & $\mathrm{~K} 7^{*}$ & 8.62 & 3.54 & 4100 & -3.39 & ARG \\
\hline $0405-0785226$ & 2MASS J18151564-4927472 & $18: 15: 15.6$ & $-49: 27: 47.3$ & M3 & 8.04 & 4.55 & 3450 & -3.16 & BPMG/ARG \\
\hline $0277-1048331$ & HD 173167 & $18: 48: 06.4$ & $-62: 13: 47.0$ & F5V & 6.14 & 1.16 & 6450 & -4.35 & $\mathrm{BPMG} / \mathrm{ARG}$ \\
\hline 0601-0968580 & - & $18: 58: 04.7$ & $-29: 53: 32.2$ & Mo* & 8.76 & 4.08 & 3700 & -2.93 & BPMG \\
\hline 0438-0823866 & 2MASS J19233820-4606316 & $19: 23: 38.2$ & $-46: 06: 31.7$ & Mo & 8.27 & 3.77 & 3850 & -3.21 & BPMG/ARG \\
\hline $0577-1232093$ & 2MASS J20055640-3216591 & 20:05:56.4 & $-32: 16: 59.2$ & $\mathrm{M} 2^{*}$ & 7.94 & 4.44 & 3550 & -3.56 & BPMG \\
\hline 0706-0872415 & 2MASS J21100535-1919573 & $21: 10: 05.4$ & $-19: 19: 57.6$ & M2 & 7.20 & 4.84 & 3350 & -2.96 & BPMG \\
\hline
\end{tabular}

stars we obtained high-resolution spectra using two different instruments.

Nine spectra were obtained in 2009 July and August with the Echelle spectrograph at the 2.3-m telescope of the Australian National University (Siding Spring Observatory, Australia). The spectra covered the whole visual range in 27
Echelle orders between $3900 \AA$ and $6720 \AA$ with only small gaps between the three reddest orders. The nominal spectral resolution is $\lambda / \Delta \lambda \approx 23000$ at the $\mathrm{H} \alpha$ line $(6563 \AA)$. The integration time per object ranged from $180 \mathrm{~s}$ to $1800 \mathrm{~s}$, depending on the brightness, and we achieved signal-to-noise ratios of about 100 around the $\mathrm{H} \alpha$ line. All data were re- 
Table 2. Additional properties and membership status of the candidate members. (1) NOMAD-identifier. (2) Reference for source spectroscopic information. ANU and FEROS refer to our observations with the Echelle spectrograph at the 2.3-m telescope of the Australian National University and with the FEROS instrument at the $2.2 \mathrm{~m}$ MPG/ESO telescope, respectively. SACY: spectroscopic data taken from Torres et al. (2006). (3) Heliocentric radial velocity. (4) Measured lithium equivalent width. (5) Measured $\mathrm{H}_{\alpha}$ equivalent width (if the line is in emission). (6) Spectroscopic binary candidates. (7) $\Delta M_{K s}$ values (for definition see Sect. 2.1) for all relevant kinematic groups listed in (12). (8) Derived $\chi_{r}^{2}$ values for all relevant kinematic groups. (9) Derived $d_{U V W}$ values for all relevant kinematic groups. (10) Membership status based on the kinematic criteria described in Sect.4.1.1] '1' or '0' indicates whether the criterion is fulfilled or not. (11) Membership status based on the lithium content. '1' or '0': the criterion is fulfilled or not, '-': lithium content cannot be used as a reliable parameter to judge the membership status (see more in Sect. 4.1.2) (12) Identifiers of candidate moving group(s).

\begin{tabular}{|c|c|c|c|c|c|c|c|c|c|c|c|}
\hline $\begin{array}{l}\text { NOMAD ID } \\
(1)\end{array}$ & $\begin{array}{l}\text { Ref. } \\
(2)\end{array}$ & $\begin{array}{r}\mathrm{RV} \\
{\left[\mathrm{km} \mathrm{s}^{-1}\right]} \\
(3)\end{array}$ & $\begin{array}{r}\mathrm{EW}_{\mathrm{Li}} \\
{[\mathrm{m} \AA]} \\
(4)\end{array}$ & $\begin{array}{r}\mathrm{EW}_{\mathrm{H}_{\alpha}} \\
{[\AA]} \\
(5)\end{array}$ & $\begin{array}{l}\text { SB? } \\
(6)\end{array}$ & $\begin{array}{r}\Delta \mathrm{M}_{K s} \\
{[\mathrm{mag}]} \\
(7)\end{array}$ & $\begin{array}{l}\chi_{r}^{2} \\
(8)\end{array}$ & $\begin{array}{r}d_{U V W} W \\
{\left[\mathrm{~km} \mathrm{~s}^{-1}\right]} \\
(9)\end{array}$ & $\begin{array}{l}\mathrm{C}_{\mathrm{kin}} \\
(10)\end{array}$ & $\begin{array}{l}\mathrm{C}_{\mathrm{Li}} \\
(11)\end{array}$ & $\begin{array}{r}\text { Candidate groups } \\
\qquad(12)\end{array}$ \\
\hline 0367-0004557 & $\mathrm{ANU}$ & $+9.4 \pm 0.8$ & $105 \pm 12$ & - & - & $-0.18 /-0.18 /-0.03$ & $1.3 / 7.3 / 17.1$ & $2.1 / 5.6 / 11.1$ & $1 / 0 / 0$ & $1 / 1 / 1$ & $\mathrm{COL} / \mathrm{THA} / \mathrm{ABD}$ \\
\hline 0361-0008966 & $\mathrm{ANU}$ & $+11.5 \pm 1.6$ & $146 \pm 8$ & - & - & -0.39 & 0.7 & 2.7 & 1 & 1 & $\mathrm{COL}$ \\
\hline $0540-0027893$ & $\mathrm{ANU}$ & $+18.8 \pm 0.8$ & $240 \pm 14$ & - & - & -0.13 & 0.01 & 0.3 & 1 & 1 & $\mathrm{COL}$ \\
\hline 0453-0032480 & ANU & $+19.5 \pm 2.3$ & $160 \pm 14$ & - & - & +0.22 & 0.8 & 3.2 & 1 & 1 & $\mathrm{COL}$ \\
\hline 0454-0034540 & $\mathrm{SACY}$ & $+19.3 \pm 0.2$ & 285 & - & - & +0.45 & 2.0 & 3.6 & 1 & 1 & $\mathrm{COL}$ \\
\hline 0466-0044706 & $\mathrm{ANU}$ & $+23.6 \pm 1.8$ & $158 \pm 10$ & - & - & +0.55 & 0.3 & 1.5 & 1 & 1 & $\mathrm{COL}$ \\
\hline 0466-0044732 & $\mathrm{ANU}$ & $+22.6 \pm 6.7$ & $<41$ & - & - & +0.01 & 0.2 & 2.2 & 1 & - & $\mathrm{COL}$ \\
\hline 0211-0114754 & SACY & $+18.7 \pm 1.0$ & 226 & - & - & -0.20 & 1.7 & 3.5 & 1 & 1 & $\mathrm{COL}$ \\
\hline 0298-0039269 & ANU & $+26.6 \pm 1.2$ & $110 \pm 9$ & - & - & -0.43 & 5.2 & 5.3 & 0 & 1 & $\mathrm{COL}$ \\
\hline 0211-0129478 & $\mathrm{SACY}$ & $+17.5 \pm 0.5$ & 265 & - & - & +0.00 & 2.4 & 3.7 & 1 & 1 & $\mathrm{COL}$ \\
\hline 0404-0048765 & FEROS & $+23.3 \pm 0.5$ & $<16$ & - & $\mathrm{Y}$ & +0.10 & 3.3 & 4.5 & 1 & 0 & ARG \\
\hline 0351-0047432 & $\mathrm{ANU}$ & $+22.5 \pm 1.0$ & $76 \pm 9$ & - & - & -0.07 & 0.2 & 1.2 & 1 & 0 & $\mathrm{COL}$ \\
\hline 0287-0048860 & $\mathrm{ANU}$ & $+22.6 \pm 1.1$ & $85 \pm 8$ & - & - & -0.10 & 0.06 & 0.6 & 1 & 1 & $\mathrm{CAR}$ \\
\hline 0339-0066782 & FEROS & $+26.1 \pm 2.6$ & $39 \pm 10$ & - & $\mathrm{Y}$ & +0.47 & 0.5 & 3.1 & 1 & 0 & $\mathrm{COL}$ \\
\hline $0315-0057897$ & FEROS & $+19.5 \pm 1.0$ & $175 \pm 10$ & - & - & +0.52 & 2.7 & 3.7 & 1 & 1 & CAR \\
\hline $0352-0070710$ & FEROS & $+19.8 \pm 0.4$ & $222 \pm 10$ & - & - & -0.04 & 2.1 & 3.5 & 1 & 1 & ARG \\
\hline 0368-0077090 & FEROS & $-10.9 \pm 8.4$ & $<26$ & - & $\mathrm{Y}$ & -0.67 & 10.9 & 34.5 & 0 & 0 & $\mathrm{COL}$ \\
\hline 0263-0084896 & FEROS & $+22.1 \pm 0.4$ & $164 \pm 10$ & - & - & $+0.36 /-0.04$ & $0.01 / 2.3$ & $0.3 / 3.0$ & $1 / 1$ & $1 / 1$ & $\mathrm{CAR} / \mathrm{COL}$ \\
\hline 0664-0176864 & FEROS & $+24.7 \pm 1.1$ & $<20$ & - & - & +0.43 & 0.01 & 0.3 & 1 & 0 & $\mathrm{COL}$ \\
\hline 0331-0114466 & FEROS & $+23.4 \pm 0.3$ & $228 \pm 10$ & - & - & +0.31 & 0.4 & 1.6 & 1 & 1 & CAR \\
\hline 0406-0105889 & FEROS & $+24.3 \pm 0.3$ & $219 \pm 11$ & - & - & +0.29 & 0.7 & 1.8 & 1 & 1 & COL \\
\hline 0248-0110675 & FEROS & $+21.7 \pm 0.4$ & $257 \pm 10$ & - & - & $+0.27 /-0.12$ & $0.03 / 1.4$ & $0.4 / 2.3$ & $1 / 1$ & $1 / 1$ & $\mathrm{CAR} / \mathrm{COL}$ \\
\hline $0248-0115043$ & FEROS & $+5.6 \pm 0.3$ & $144 \pm 10$ & - & - & $-0.26 /+0.67$ & $6.2 / 89.3$ & $5.9 / 14.6$ & $0 / 0$ & $1 / 1$ & $\mathrm{ARG} / \mathrm{COL}$ \\
\hline 0354-0106699 & FEROS & $+14.6 \pm 0.4$ & $164 \pm 10$ & - & - & +0.41 & 0.03 & 0.5 & 1 & 1 & ARG \\
\hline 0341-0108561 & FEROS & $+13.9 \pm 0.8$ & $218 \pm 11$ & $-0.20 \pm 0.06$ & - & +0.33 & 0.3 & 2.4 & 1 & 1 & ARG \\
\hline 0489-0152365 & FEROS & $+25.7 \pm 0.4$ & $117 \pm 10$ & - & - & +0.26 & 2.4 & 2.5 & 1 & 1 & $\mathrm{COL}$ \\
\hline 0269-0122275 & FEROS & $+22.1 \pm 0.7$ & $201 \pm 11$ & - & - & -0.02 & 0.10 & 0.8 & 1 & 1 & CAR \\
\hline 0291-0099052 & FEROS & $+22.3 \pm 0.4$ & $246 \pm 10$ & - & - & +0.49 & 0.8 & 2.6 & 1 & 1 & CAR \\
\hline $0372-0169783$ & FEROS & $+23.3 \pm 0.5$ & $275 \pm 11$ & $-0.38 \pm 0.10$ & - & $+0.39 /+0.94$ & $1.0 / 0.3$ & $2.0 / 1.6$ & $1 / 1$ & $1 / 1$ & $\mathrm{COL} / \mathrm{CAR}$ \\
\hline 0313-0102916 & FEROS & $+22.0 \pm 0.7$ & $274 \pm 16$ & - & - & $-0.17 /+0.29$ & $0.4 / 0.2$ & $1.2 / 1.3$ & $1 / 1$ & $1 / 1$ & $\mathrm{COL} / \mathrm{CAR}$ \\
\hline 0397-0143387 & SACY & +22.5 & 340 & - & - & $+0.92 /+0.36$ & $0.2 / 0.8$ & $1.9 / 3.0$ & $1 / 1$ & $1 / 1$ & $\mathrm{CAR} / \mathrm{COL}$ \\
\hline $0268-0125760$ & FEROS & $-10.3 \pm 1.8$ & $<20$ & - & - & $+0.02 /-0.91 /+0.42$ & $82.6 / 31.0 / 92.5$ & $30.0 / 21.1 / 31.5$ & $0 / 0 / 0$ & $0 / 0 / 0$ & COL/ARG/CAR \\
\hline $0348-0138354$ & FEROS & $+33.8 \pm 3.9$ & $252 \pm 14$ & - & $\mathrm{Y}$ & $+0.32 /-0.17$ & $3.0 / 3.8$ & $11.6 / 13.1$ & $0 / 0$ & $1 / 1$ & CAR/COL \\
\hline 0308-0116109 & FEROS & $+21.6 \pm 1.1$ & $276 \pm 15$ & - & $\mathrm{Y}$ & $+0.57 /+0.13$ & $0.01 / 0.6$ & $0.3 / 2.2$ & $1 / 1$ & $1 / 1$ & $\mathrm{CAR} / \mathrm{COL}$ \\
\hline 0337-0139009 & FEROS & $+20.0 \pm 0.3$ & $107 \pm 10$ & - & - & $-0.20 /+0.27$ & $0.2 / 2.1$ & $0.7 / 2.2$ & $1 / 1$ & $0 / 0$ & $\mathrm{COL} / \mathrm{CAR}$ \\
\hline 0415-0155444 & FEROS & $+21.7 \pm 0.4$ & $157 \pm 9$ & - & - & $+0.18 /-0.37$ & $0.6 / 0.2$ & $1.4 / 1.3$ & $1 / 1$ & $1 / 1$ & $\mathrm{CAR} / \mathrm{COL}$ \\
\hline 0193-0211246 & FEROS & $+19.4 \pm 0.4$ & $213 \pm 10$ & - & - & $+0.09 /-0.98$ & $0.02 / 14.5$ & $0.4 / 12.1$ & $1 / 0$ & $1 / 1$ & $\mathrm{CAR} / \mathrm{ARG}$ \\
\hline 0367-0179130 & FEROS & $+22.6 \pm 0.8$ & $315 \pm 10$ & $-0.15 \pm 0.03$ & - & $+0.03 /-0.45$ & $0.1 / 1.1$ & $0.7 / 2.1$ & $1 / 1$ & $1 / 1$ & $\mathrm{CAR} / \mathrm{COL}$ \\
\hline 0393-0168117 & FEROS & $+5.8 \pm 0.4$ & $277 \pm 10$ & - & - & $+0.24 /+0.73$ & $88.1 / 108.8$ & $15.0 / 16.4$ & $0 / 0$ & $1 / 1$ & $\mathrm{COL} / \mathrm{CAR}$ \\
\hline $0327-0202270$ & FEROS & $+20.4 \pm 2.3$ & $171 \pm 13$ & - & $\mathrm{Y}$ & $-0.02 /-0.44$ & $0.05 / 0.2$ & $0.9 / 1.5$ & $1 / 1$ & $1 / 1$ & $\mathrm{CAR} / \mathrm{COL}$ \\
\hline 0348-0190850 & FEROS & $+11.8 \pm 0.8$ & $37 \pm 10$ & - & - & +0.23 & 0.5 & 2.0 & 1 & 0 & ARG \\
\hline 0350-0195011 & FEROS & $+27.1 \pm 1.7$ & $21 \pm 12$ & - & $\mathrm{Y}$ & +0.17 & 3.7 & 6.4 & 0 & 0 & CAR \\
\hline 0154-0097276 & FEROS & $+17.8 \pm 0.3$ & $199 \pm 16$ & - & - & $+0.26 /-0.64$ & $0.05 / 21.7$ & $0.6 / 12.7$ & $1 / 0$ & $1 / 1$ & $\mathrm{CAR} / \mathrm{ARG}$ \\
\hline $0349-0227761$ & FEROS & $+6.2 \pm 2.0$ & $<35$ & $-0.74 \pm 0.05$ & $\mathrm{Y}$ & +0.63 & 0.2 & 1.8 & 1 & 0 & ARG \\
\hline $0277-0339323$ & FEROS & $+43.4 \pm 1.6$ & $50 \pm 14$ & $-1.23 \pm 0.10$ & - & $+0.51 /+0.82 /-0.27$ & $79.9 / 73.6 / 113.6$ & $27.2 / 25.1 / 37.2$ & $0 / 0 / 0$ & $0 / 0 / 1$ & $\mathrm{COL} / \mathrm{CAR} / \mathrm{ARG}$ \\
\hline $0370-0341486$ & FEROS & $+5.1 \pm 1.0$ & $43 \pm 11$ & $-0.97 \pm 0.12$ & - & $-0.02 /+0.74$ & $0.04 / 19.3$ & $0.5 / 10.7$ & $1 / 0$ & $1 / 1$ & $\mathrm{ARG} / \mathrm{COL}$ \\
\hline 0104-0056948 & FEROS & $+22.6 \pm 0.5$ & $108 \pm 11$ & 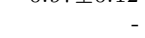 & - & $+0.17 /+0.65$ & $47.5 / 1.1$ & $20.8 / 3.1$ & $0 / 1$ & $0 / 1$ & $\mathrm{ARG} / \mathrm{ABD}$ \\
\hline $0568-0654078$ & FEROS & $+29.4 \pm 1.5$ & $<27$ & - & - & +0.46 & 317.8 & 49.5 & 0 & 0 & ARG \\
\hline $0405-0785226$ & FEROS & $-7.7 \pm 6.1$ & $<46$ & $-5.00 \pm 0.20$ & $\mathrm{Y}$ & $-0.34 /-1.00$ & $0.4 / 1.5$ & $4.8 / 8.1$ & $1 / 0$ & - & BPMG/ARG \\
\hline $0277-1048331$ & FEROS & $+0.8 \pm 7.0$ & $107 \pm 16$ & $\frac{-10000}{-}$ & $\mathrm{Y}$ & $+0.15 /-0.97$ & $0.03 / 2.1$ & $1.1 / 11.0$ & $1 / 0$ & $1 / 1$ & BPMG/ARG \\
\hline 0601-0968580 & FEROS & $-7.0 \pm 0.3$ & $<28$ & $-4.90 \pm 0.20$ & - & +0.08 & 0.3 & 2.2 & 1 & - & BPMG \\
\hline $0438-0823866$ & FEROS & $-0.2 \pm 1.2$ & $422 \pm 12$ & $-1.13 \pm 0.15$ & - & $+0.04 /-0.66$ & $0.7 / 46.4$ & $3.1 / 15.7$ & $1 / 0$ & $1 / 1$ & BPMG/ARG \\
\hline $0577-1232093$ & FEROS & $-5.1 \pm 1.3$ & $140 \pm 16$ & $-1.92 \pm 0.15$ & - & -0.09 & 0.4 & 1.7 & 1 & 1 & BPMG \\
\hline $0706-0872415$ & FEROS & $-6.3 \pm 1.2$ & $<41$ & $-3.21 \pm 0.15$ & - & -0.12 & 0.3 & 1.3 & 1 & - & BPMG \\
\hline
\end{tabular}

duced with standard IRAF1 tasks, including bias and flat-

1 IRAF is distributed by the National Optical Astronomy Observatories, which are operated by the Association of Universities for Research in Astronomy, Inc., under cooperative agreement with the National Science Foundation. field corrections, cosmic ray removal, extraction of the 27 individual orders of the echelle spectra, wavelength calibration, and continuum normalization. ThAr spectral lamp exposures were regularly taken before and after every object spectrum to monitor the wavelength shifts of the spectra on the CCD.

41 additional targets were observed using the FEROS 
instrument (the Fiber-fed Extended Range Optical Spectrograph, Kaufer et al. 1999) mounted at the $2.2 \mathrm{~m} \mathrm{MPG/ESO}$ telescope in La Silla, Chile, between 2011 April 15 and 18. FEROS is an Échelle spectrograph that covers the spectral region between 3500 and $9200 \AA$ in 39 echelle orders in one exposure, providing a spectral resolution of $R \approx 48000$. We used the "object-sky" mode in our observations in which one of the two fibers is positioned at the target while the other one observes the sky. The integration times ranged between $180 \mathrm{~s}$ and $3200 \mathrm{~s}$ depending on the target brightness. The resulting signal-to-noise ratio was $\gtrsim 60$ at the lithium line (Li I, $6708 \AA$ ). Standard calibration observations, including bias, dark, and flat exposures, as well as Th-Ar lamp calibration spectra, were obtained at each night. The data were processed using the FEROS data reduction system (DRS) provided by ESO. The reduction procedure includes bias subtraction, flat-field correction, background subtraction, the definition and extraction of orders, and wavelength calibration.

\subsection{Obtaining the radial velocities (RVs)}

All spectra were evaluated according to the standard fashion in IRAF. Template spectra were taken from Munari et al. (2005). In the velocity fitting procedure we selected those models that provided the closest effective temperature to that derived from the photometry and that had $[\mathrm{Fe} / \mathrm{H}]=0$, $v \sin i=0, \log g=4.5$. Cross correlation functions (CCFs) were calculated by the FXCOR task in IRAF, in the 5200-6400 $\mathrm{K}$ range. Continuum normalization is a difficult task in this spectral range, especially for late-type stars, mostly because of the presence of molecular bands. Both observed and model spectra were normalized to a 13 th order polinomial model. This model likely clipped the widest bands, too, but resulted in a satisfactorily smooth correlation function, unaffected by the misestimation of the band shapes.

During the whole task, the most challenging problem was the crude temperature resolution of model spectrum libraries, and in many cases, template and observed spectra exhibited prominent differences in the continuum/molecular band level. We tested how the misestimation of the template spectra affects the radial velocity determination. The test led to statisfactory results: although the calculated error of the RV increased a lot with non-adequate template spectra, the estimated RV value did not change significantly. Therefore we are confident of the reliability of the fitted RVs, however, we have to note that a considerable part of the errors may have come from the lack of a template spectrum with appropriate temperature. The resulting RVs are listed in Table 2

\subsection{Identification of spectroscopic binaries}

Candidate spectroscopic binaries were identified by complex structures in the CCF, in most cases two maxima could be found with similar heights. In some cases, the second maximum was not observed, but a prominent skewness of the $\mathrm{CCF}$, or a plateau on the wings appeared, likely due to a companion. For these targets the CCF were fitted manually assuming two stars, and we derived the velocities and the velocity differences. (In this paper, we use the velocity derived for the brighter component, if binarity was suspected.)
The calculated velocity differences were typically in the 20-50 $\mathrm{km} \mathrm{s}^{-1}$ range, which is compatible with orbital periods of several 10 days to a year, although this is not an exact estimate because we only have velocity amplitudes from one measurement. In the case of 0368-0077090, the binarity was confirmed by the variation of the radial velocity of the brighter component, as observations taken at different epochs led to radial velocities that were incompatible with a constant value.

\subsection{Measuring the equivalent width of the $\mathrm{Li} \mathrm{I}$ and $\mathbf{H}_{\alpha}$ lines}

We used the IRAF continuum task with a cubic spline algorithm to model the local continuum in the wavelength ranges between 6680 and $6736 \AA$ and between 6520 and $6605 \AA$ around the lithium and $\mathrm{H}_{\alpha}$ lines, respectively, normalizing all fluxes to unity. The equivalent widths of lithium absorption lines $\left(\mathrm{EW}_{\mathrm{Li}}\right)$ were measured by fitting Gaussian profiles using a self-developed IDL routine. A measurable amount of $\mathrm{Li}$ has been detected in the spectra of 40 stars. For the rest of the sample (10 targets), we estimated upper limits for the equivalent widths using our code. Equivalent widths of the $\mathrm{H}_{\alpha}$ line were also obtained for stars where this line was in emission. The measured $\mathrm{Li}$ I and $\mathrm{H}_{\alpha}$ equivalent widths are listed in Table 2 .

\section{RESULTS}

\subsection{Assignment decision}

Members of young moving groups can be identified based on their common space motion within the Galaxy and their common ages. Supplementing astrometric data by new radial velocity measurements, the similarity between the Galactic space motion of the candidate stars and characteristic space motion of groups can be evaluated more reliably than based only on proper motion data (especially in those cases where measured trigonometric parallaxes are also available). As a preliminary age diagnostic in the sample selection, we kept only those candidates that exhibited coronal activity similar to the known member of young moving groups and whose position in the colour-magnitude diagram coincided well with the loci of the known members (Fig. 1). By comparing the measured Li contents with the Li distribution of the specific association(s) we can investigate further whether our targets could be co-eval with the appropriate group.

\subsubsection{Kinematic criteria}

Using the new radial velocity data we computed the Galactic space velocity for each candidate star. We used a righthanded UVW coordinate system ( $\mathrm{U}$ is positive towards the Galactic centre, $\mathrm{V}$ is positive in the direction of galactic rotation and $\mathrm{W}$ is positive towards the North Galactic pole) and followed the general recipe described in "The Hipparcos and Tycho Catalogues" (ESA 1997). Coordinates and proper motions of stars were taken from the NOMAD catalogue. For targets included in the Hipparcos catalogue we used their measured trigonometric parallaxes, otherwise we adopted 


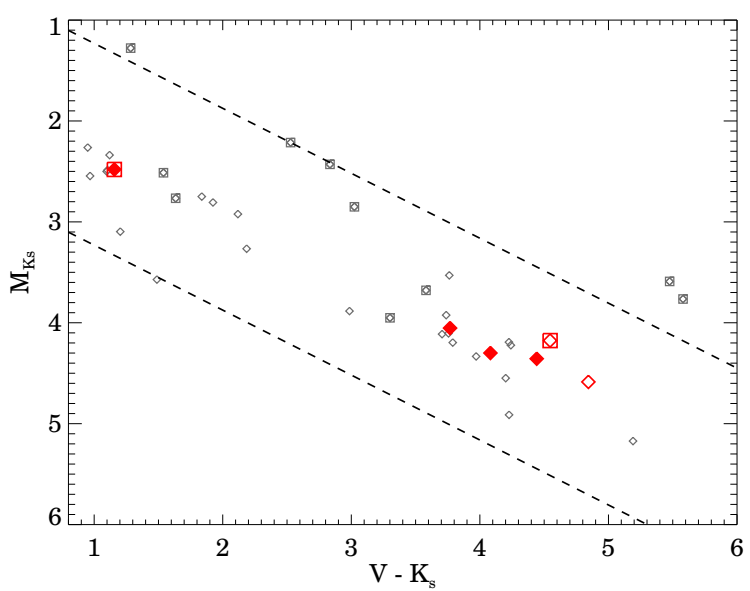

Figure 1. Absolute $K_{\mathrm{S}}$ magnitude versus $\mathrm{V}-\mathrm{K}_{\mathrm{S}}$ colour diagram for the known and candidate members of BPMG. Known members are displayed by small grey diamonds. Larger black (red in the electronic version) diamonds represent our candidate stars taken from Table 1 Squares denote those close binaries for which no photometric data are available separately for the individual components. Following the outline of Lépine \& Simon (2009) the loci of the known members of the specific groups were fitted with a linear, the \pm 1 magnitude range of the fit is represented by dashed lines. Nearly all known members are located within these lines in the diagram. Candidate objects outside this area were omitted from our survey (see Sect. 2.1). Those candidates that are finally classified as likely members of BPMG are marked by filled symbols.

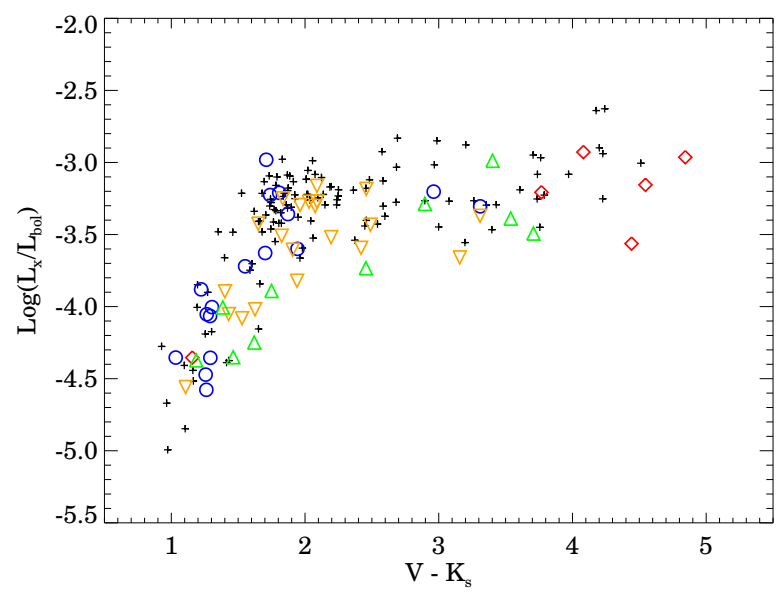

Figure 2. Fractional X-ray luminosities as a function of $\mathrm{V}-\mathrm{K}_{\mathrm{S}}$ for the known and candidate members of the five kinematic groups. Plus signs represent the previously known members of the groups. Candidate members of BPMG, COL, CAR and ARG are taken from Table 1 and marked by diamonds (red in the electronic version), circles (blue in the electronic version), upside down triangles (orange in the electronic version) and triangles (green in the electronic version), respectively.

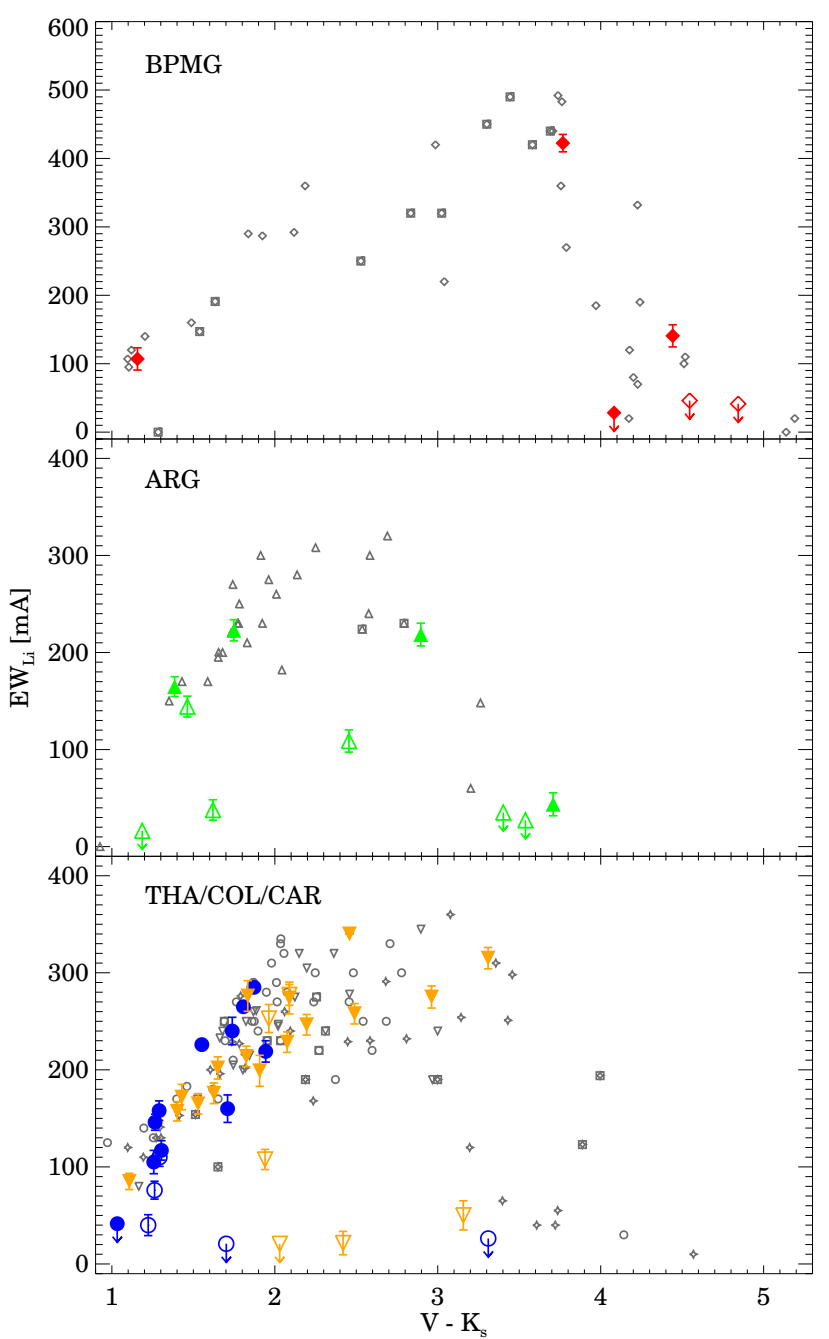

Figure 3. Equivalent width of lithium as a function of $\mathrm{V}-\mathrm{K}_{\mathrm{s}}$. Candidate members of BPMG, ARG, COL and CAR are marked by diamonds (red in the electronic version), triangles (green in the electronic version), circles (blue in the electronic version) and upside-down triangles (orange in the electronic version), respectively. Known members of a specific group are represented by similar but smaller grey symbols than the candidates. Known THA members are displayed by small grey star symbols. Candidates that turned out to be likely members of a specific group are marked by filled symbols.

the derived kinematic parallaxes. Radial velocity data were taken from Table2 For 0466-0044732 (HIP 25436) and 04660044706 (HIP 25434), which constitute a binary system according to the The Washington Visual Double Star Catalog (Mason et al. 2001), we used the weighted average of their measured trigonometric parallaxes.

In order to characterize the kinematic match between the candidates and different groups, we followed Shkolnik et al. 2012) by defining two parameters: (1) a velocity modulus $d_{U V W}=$ $\sqrt{\left.\left(U-U_{0}\right)^{2}+\left(V-V_{0}\right)^{2}+\left(W-W_{0}\right)^{2}\right)}$ that shows the distance of the measured $U, V, W$ components to the characteristic space motion of the different moving groups $\left(U_{0}, V_{0}, W_{0}\right)$, and (2) a reduced $\chi^{2}$ statistics with 3 degrees of 


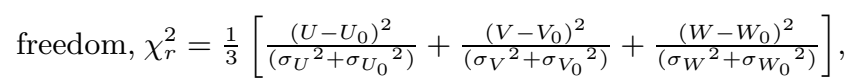
where $\sigma_{U}, \sigma_{V}, \sigma_{W}$ are the uncertainties of the measured $U, V, W$ velocities of the star, while $\sigma_{U_{0}}, \sigma_{V_{0}}, \sigma_{W_{0}}$ are the uncertainties of characteristic space motion of the specific moving group (taken from Torres et al. 2008). We computed and listed these values in Table 2 for each star with respect to each relevant group. We also checked the resulting $d_{U V W}$ and $\chi_{r}^{2}$ values for objects already assigned to a group in the literature. In accordance with Shkolnik et al. (2012), for most of the known members $(\gtrsim 90 \%)$ we obtained $d_{U V W}<5 \mathrm{~km} \mathrm{~s}^{-1}$ and $\chi_{r}^{2}<6$, therefore we set these values as a lower limit of membership for the new candidates as well. Membership status of our targets, derived from these kinematic criteria $\left(\mathrm{C}_{k i n}\right)$, is reviewed in Table 2. We note that in some cases the target could be assigned to several different moving groups but the new radial velocity data helped to constrain the assignment for one specific group (e.g. 0367-0004557).

In the case of candidate spectroscopic binaries with double peak in the correlation function we included the RV of the brighter peak in the analysis (see Sect. 3.3). Therefore, $\mathrm{RV}$ values of these systems are biased estimates of the systemic velocity, with currently unknown bias values that may reach even $10 \mathrm{~km} \mathrm{~s}^{-1}$ in some cases. There are also candidate spectroscopic binaries where the correlation function had significant skewness, but exhibited only a single prominent peak, which gives also a biased estimate of the systemic velocity. The proper disentangling of the components will require higher resolution, higher signal-to-noise ratio, and multi-epoch observations. This approach makes the assignment of the specific objects less reliable and can lead to the rejection/acceptance of a real/false group member.

\subsubsection{Membership status based on lithium content}

Due to the fact that lithium is destroyed at relatively low temperatures $\left(2.5 \times 10^{6} \mathrm{~K}\right)$ in stellar interiors, the primordial lithium depletes with time in stars with a convective layer, that makes this element one of the best age indicators for young stars (Zuckerman \& Song 2004). We used the equivalent width of the lithium line $\left(\mathrm{EW}_{\mathrm{Li}}\right)$ as an empirical measurement of the lithium abundances in our targets. In accordance with our general principle, for membership of a candidate we requested that its lithium content is consistent with those of known members. In Fig. 3 we displayed the measured $\mathrm{EW}_{\mathrm{Li}}$ values of our candidates and that of known members of specific groups, as a function of $V-K_{s}$ colour indices. Members and member candidates of THA, COL, and CAR groups are plotted together since from the $\mathrm{Li}$ abundance point of view these three groups are practically indistinguishable (da Silva et al. 2009). In order to evaluate whether the $\mathrm{Li}$ content of a certain candidate is consistent with the $\mathrm{Li}$ distribution of the assigned group, we compared the target's measured $\mathrm{EW}_{\mathrm{Li}}$ to that of known members with similar $V-K_{s}$ indices. For each target we selected those known members of the assigned group for comparison whose $V-K_{s}$ indices are within a $\pm r_{V-K_{s}}$ magnitude range of the candidates star's $\left(V-K_{s}\right)_{c}, r_{V-K_{s}}$ was set to $0.07 \mathrm{mag}$ if $\left(V-K_{s}\right)_{c}<3 \mathrm{mag}$ and $0.1 \mathrm{mag}$ if $\left(V-K_{s}\right)_{c} \geqslant 3$ mag. A candidate was accepted as a member - in terms of lithium abundance - if its $\mathrm{EW}_{\mathrm{Li}} \pm \sigma_{E W_{L i}}$ value falls in the $\mathrm{EW}_{\mathrm{Li}}$ range of the selected known members. For some ARG candidate stars where we could not find any known members within the abovementioned colour index range, we used GAYA (THA/COL/CAR) stars - whose age is close to that of ARG - for this comparison. The rate of lithium depletion depends also on the effective stellar temperature. In early M-type stars the Li can be entirely depleted by an age of 10 Myr (Zuckerman \& Song 2004; Mentuch et al. 2008), thus the lack of lithium in the spectrum of such stars cannot be used to unambigously reject the membership in groups with ages $>10$ Myr.

Membership status of our targets, derived based on this criterion $\left(\mathrm{C}_{L i}\right)$, is listed in Table 2 .

\subsubsection{Final membership}

For the final membership we demanded that both our kinematic and lithium content based criteria are fulfilled $\left(\mathrm{C}_{k i n}\right.$ and $\left.\mathrm{C}_{L i}=1\right)$. This means that taking into account our preliminary selection criteria, our targets have comparable X-ray luminosity and lithium abundance than those of the known members with similar colour indices, their position in the colour-magnitude diagram are consistent with the loci of known members, and their Galactic space motion and location are also consistent with the characteristic space motion and position of the assigned group. According to our classification, two M-type stars (0405-0785226 and 0706-0872415) for which the lithium-based diagnostic method cannot be used to support or reject the membership status were not adopted as probable new members of BPMG. 0466-0044732 and 0601-0968580, whose lithium content was also not relevant in the classification, were approved as probable new members because they are located in binary systems where the other companion is considered as probable member of the specific group (see Sect. 4.3). There are three candidate binary systems (0308-0116109, 0327-0202270, and 0277-1048331) which fulfilled both the kinematic and lithium content based criteria. Since RV values of these systems can only be considered as biased estimates of the systemic radial velocity, their $\mathrm{U}, \mathrm{V}, \mathrm{W}$ values are less reliable and further observations are needed to finally consolidate our assigments. Therefore, 0308-0116109 and 0327-0202270 were labeled as possible members only. Despite the binarity issue, 0277-1048331 was classified as a probable member because it has a widely separated comoving pair (TYC 9073-0762, for details see Sect.4.3) that is found to be a member of the BPMG by Torres et al. (2008). As a result of our survey, we identified 33 new probable and 2 new possible members (see in Table 3) of four young nearby moving groups (BPMG, COL, CAR, ARG). All probable members have $d_{U V W}<3.73 \mathrm{~km} \mathrm{~s}^{-1}$, while the largest $\chi_{r}^{2}$ is 2.69. Assuming that the derived $\chi_{r}^{2}$ values follow a $\chi^{2}$ distribution with three degrees of freedom (see also Shkolnik et al. 2012) the kinematic membership probability corresponding to $\chi_{r}^{2}=2.69$ is 0.44 . Thus, all 33 selected stars exhibit a membership probability of at least $44 \%$, a strong indication of membership.

There are several targets in our candidate list that can be assigned to more than one kinematic groups. In some cases, our new RV and Li content-based analysis helped to resolve this ambiguity leaving only one potential group (e.g. 0367-0004557). In eight cases (0263-0084896, 0248-0110675, 
0372-0169783, 0313-0102916, 0397-0143387, 0308-0116109, 0415-0155444, 0367-0179130), however, the ambiguity remained. These objects can either be assigned to the Columba or the Carina moving groups. These two groups have very similar space motion and somewhat overlapping spatial location. In order to establish the more probable group for these targets, we compared 1) their distances to the mean position of the COL and CAR groups $\left(d_{X Y Z}\right.$, mean $\mathrm{X}, \mathrm{Y}, \mathrm{Z}$ positions were taken from, Torres et al. 2008) and 2) their $d_{U V W}$ and $\chi_{r}^{2}$ values were computed for the two relevant groups. For seven ambiguous targets (0263-0084896, 02480110675, 0372-0169783, 0313-0102916, 0397-0143387, 03080116109, 0367-0179130) the assigment to the Carina association is more likely based on these parameters, because $d_{U V W}^{C A R} \leqslant d_{U V W}^{C O L}, \chi_{r, C A R}^{2} \leqslant \chi_{r, C O L}^{2}$ and $d_{X Y Z}^{C A R}<d_{X Y Z}^{C O L}$. The case of 0415-0155444 is somewhat more difficult because its space motion matches the values of COL $\left(d_{U V W}^{C O L}=\right.$ $1.26 \mathrm{~km} \mathrm{~s}^{-1}, \chi_{r, C O L}^{2}=0.24$ while $d_{U V W}^{C A R}=1.38 \mathrm{~km} \mathrm{~s}^{-1}$, $\left.\chi_{r, C A R}^{2}=0.6\right)$ better, however, the star is located significantly closer to the mean position of CAR $\left(d_{X Y Z}^{C A R}=22.2 \mathrm{pc}\right.$, while $d_{X Y Z}^{C O L}=93.0 \mathrm{pc}$ ). This star was finally assigned also to the Carina group due to the better match in the space position.

Trigonometric parallaxes measured by Hipparcos are available only for 4 stars. These targets can be considered as new secure young moving group members. For the remaining 31 stars, we applied kinematic distances computed based on the assumption that they belong to the specific kinematic group. By deriving kinematic distances for the abovementioned four secure members, as well as for all those previously reported members of the selected groups for which Hipparcos data were also available (taken from Torres et al. 2008) we found that the kinematic and trigonometric parallaxes agree within the $2 \sigma$ uncertainties for nearly all targets (and within $1 \sigma$ for $75 \%$ of the sample). Despite this good agreement and also that kinematic and photometric distances of the 31 stars are consistent, we cannot completely exclude that their real distance in fact deviates from the predicted one, thus their kinematic properties do not match those of the moving group. Based on the present data, these 31 stars can be classified as probable (29 cases) or possible (2 objects) new members, however, these objects yet lack trigonometric parallaxes, and further observations (e.g. by the Gaia mission) are necessary to prove our membership assignment.

In summary, we identified 4 secure members of the COL group, and suggested 31 further probable/possible members of various nearby groups. We assign 4 stars to the BPMG, 4 stars to the ARG group, 11 stars to COL, and 16 stars to CAR.

Interestingly, our observations revealed that the measured parameters of 0104-0056948 fulfill all the criteria of ABD membership, meaning that we serendipitiously identified a new probable AB Doradus member as well. Kinematic properties of this star were also listed in Table 3

There are four stars in our sample (0454-0034540, 02110114754, 0211-0129478, 0397-0143387) whose spectroscopic data were taken from the SACY survey (Torres et al. 2008). In our analysis we concluded that these stars are likely members of the Columba/Carina groups, while Torres et al. (2008) rejected them as a member of any of the five groups studied in our work. The different conclusions about group membership can be explained by the difference of the applied candidate selection algorithm and - in the case of 03970143387 - by the difference of the utilized astrometric data. Namely, for $0454-0034540$ the $d_{U V W}$ value of $3.6 \mathrm{kms}^{-1}$ is one of the highest among our sample. Torres et al. (2008) may have rejected this object due to their more strict kinematic criterion $\left(\sqrt{\left(U-U_{0}\right)^{2}+\left(V-V_{0}\right)^{2}+\left(W-W_{0}\right)^{2}}<\right.$ $\left.3.5 \mathrm{kms}^{-1}\right)$. The $\mathrm{X}$ space coordinate of 0211-0114754 and 0211-0129478 is out of the boundaries of the Columba group specified by Torres et al. (2008, in their Table 2). In the case of 0397-0143387, the likely erroneous TYCHO-based proper motions may have led to the rejection of the candidate (for more details, see Sect. 4.3).

\subsection{Search for accretion signatures in late-type candidates}

A few late-type members of the youngest nearby moving groups are still accreting (Jayawardhana et al. 2006). Therefore, we investigated whether any of our candidates show signatures of accretion. In the spectra of eleven stars, the $\mathrm{H}_{\alpha}$ line was detected in emission. Barrado y Navascués \& Martín (2003) proposed an empirical criterion to distinguish between stars with chromospheric activity and objects with accretion based on their measured $\mathrm{H}_{\alpha}$ emission. White \& Basri (2003) used the full width of this line at $10 \%$ of the peak to identify "accretors" by setting a threshold value of $270 \mathrm{~km} \mathrm{~s}^{-1}$ for this parameter. Using these indicators, we concluded that none of our targets show accretion signature and their measured relatively weak $\mathrm{H}_{\alpha}$ emission may be of chromospheric origin.

\subsection{Notes on individual targets}

\subsubsection{New members, probable new members}

0367-0004557 (HD 3888) and 0361-0008966 (HD 8077): Both stars have trigonometric parallaxes in the Hipparcos catalogue. Their Galactic space motion is consistent with the characteristic space motion of COL. Since their high fractional X-ray luminosity and lithium content are also consistent with the respective properties of known members, we classified them as new secure members of the Columba moving group. With its distance of $\sim 45 \mathrm{pc}$, 0367-0004557 is one of the closest members of COL.

0540-0027893 (CD-36 1289): From our analysis, this F8-type star seems to be a probable member of COL. We also found that $0540-0027893$ is a co-moving pair of HD 21423 and HD 21434 with a separation of $85^{\prime \prime}$ and $107^{\prime \prime}$. The proper motion of 0540-0027893 $\left(\mu_{\alpha \cos \delta}=37.8 \pm\right.$ 1.4 mas yr$^{-1}, \mu_{\delta}=-3.6 \pm 1.4$ mas yr$^{-1}$, NOMAD) is in good agreement with the corresponding astrometric properties of HD 21423 and HD 21434, $\mu_{\alpha \cos \delta}=36.3 \pm 0.5$ mas $\mathrm{yr}^{-1}$, $\mu_{\delta}=-4.7 \pm 0.5 \operatorname{mas~yr}^{-1}$ and $\mu_{\alpha \cos \delta}=34.2 \pm 1.1 \mathrm{mas} \mathrm{yr}^{-1}$, $\mu_{\delta}=-5.0 \pm 1.1 \mathrm{mas} \mathrm{yr}^{-1}$, respectively (also from NOMAD). Grenier et al. (1999) obtained a radial velocity of $18.68 \mathrm{~km} \mathrm{~s}^{-1}$ for HD 21423 which is identical to the measured RV of $18.8 \pm 0.8 \mathrm{~km} \mathrm{~s}^{-1}$ for our target. The Hipparcos parallax of HD 21423 also matches perfectly the kinematic parallax of $0540-0027893\left(9.27 \pm 0.42{\text { mas } \mathrm{yr}^{-1} \text { and }}^{-1}\right.$ $10.06 \pm 0.72$ mas $\mathrm{yr}^{-1}$, respectively). By recomputing the 
Table 3. Final list of probable and possible new members. (1) NOMAD-identifier. (2) Parallax. Asterisks indicate parallaxes taken from van Leeuwen (2007), otherwise kinematic parallaxes (see Sect. 2.1) are listed. For 0466-0044732 and 0466-0044706 that form a binary system (see Sect. 4.1.1) we listed the weighted average of their measured trigonometric parallaxes. (3) $\Delta M_{K s}$ values. (4)-(6) Galactic space velocity components $(\mathrm{U}, \mathrm{V}, \mathrm{W})$ of the star. (7)-(8) Derived $\chi_{r}^{2}$ and $d_{U V W}$ values for the assigned moving group. For a detailed definition of these parameters see Sect. 4.1.1 (9) Multiplicity. (10) Identifier of the assigned moving group. Asterisks indicate those candidate spectroscopic binary systems that are classified only as possible members in our work (see Sect. 4.1 .3 for details).

\begin{tabular}{|c|c|c|c|c|c|c|c|c|c|}
\hline $\begin{array}{c}\text { NOMAD ID } \\
(1)\end{array}$ & $\begin{array}{r}\pi \\
{[\mathrm{mas}]} \\
(2)\end{array}$ & $\begin{array}{r}\Delta \mathrm{M}_{K s} \\
{[\mathrm{mag}]} \\
(3)\end{array}$ & $\begin{array}{r}\mathrm{U} \\
{\left[\mathrm{km} \mathrm{s}^{-1}\right]} \\
(4)\end{array}$ & $\begin{array}{r}\mathrm{V} \\
{\left[\mathrm{km} \mathrm{s}^{-1}\right]} \\
(5)\end{array}$ & $\begin{array}{r}\mathrm{W} \\
{\left[\mathrm{km} \mathrm{s}^{-1}\right]} \\
(6)\end{array}$ & $\begin{array}{l}\chi_{r}^{2} \\
(7)\end{array}$ & $\begin{array}{r}d_{U V W} \\
{\left[\mathrm{~km} \mathrm{~s}^{-1}\right]} \\
(8)\end{array}$ & $\begin{array}{c}\text { Multiplicity } \\
(9)\end{array}$ & $\begin{array}{c}\text { Group } \\
(10)\end{array}$ \\
\hline 0367-0004557 & $22.30 \pm 0.60^{*}$ & -0.18 & $-13.3 \pm 0.5$ & $-23.7 \pm 0.6$ & $-5.0 \pm 0.7$ & 1.32 & 2.12 & $\mathrm{~N}$ & $\mathrm{COL}$ \\
\hline 0361-0008966 & $10.17 \pm 0.85^{*}$ & -0.39 & $-12.3 \pm 1.3$ & $-23.8 \pm 1.8$ & $-4.3 \pm 1.5$ & 0.65 & 2.70 & $\mathrm{~N}$ & $\mathrm{COL}$ \\
\hline $0540-0027893$ & $10.06 \pm 0.72$ & -0.13 & $-13.1 \pm 0.9$ & $-21.6 \pm 1.2$ & $-5.6 \pm 1.1$ & 0.01 & 0.31 & $\mathrm{Y}$ & $\mathrm{COL}$ \\
\hline 0453-0032480 & $9.88 \pm 0.81$ & 0.22 & $-10.5 \pm 1.5$ & $-22.1 \pm 1.8$ & $-4.2 \pm 1.9$ & 0.77 & 3.17 & $\mathrm{~N}$ & $\mathrm{COL}$ \\
\hline 0454-0034540 & $11.03 \pm 0.94$ & 0.45 & $-10.5 \pm 0.7$ & $-21.3 \pm 0.8$ & $-3.6 \pm 0.9$ & 2.01 & 3.60 & $\mathrm{~N}$ & COL \\
\hline 0466-0044706 & $14.09 \pm 1.40^{*}$ & 0.55 & $-11.7 \pm 0.9$ & $-21.7 \pm 1.5$ & $-5.8 \pm 1.3$ & 0.31 & 1.53 & $\mathrm{Y}$ & COL \\
\hline 0466-0044732 & $14.09 \pm 1.40^{*}$ & 0.01 & $-11.6 \pm 2.2$ & $-20.3 \pm 5.3$ & $-5.9 \pm 3.8$ & 0.16 & 2.19 & $\mathrm{Y}$ & COL \\
\hline 0211-0114754 & $9.65 \pm 0.64$ & -0.20 & $-11.7 \pm 1.1$ & $-22.5 \pm 1.0$ & $-2.9 \pm 0.9$ & 1.73 & 3.46 & $\mathrm{~N}$ & $\mathrm{COL}$ \\
\hline $0211-0129478$ & $9.68 \pm 0.65$ & 0.00 & $-12.1 \pm 1.2$ & $-21.3 \pm 0.7$ & $-2.4 \pm 0.7$ & 2.35 & 3.73 & $\mathrm{~N}$ & $\mathrm{COL}$ \\
\hline 0287-0048860 & $12.46 \pm 1.24$ & -0.10 & $-9.9 \pm 1.1$ & $-23.4 \pm 1.1$ & $-4.2 \pm 0.9$ & 0.06 & 0.56 & $\mathrm{~N}$ & CAR \\
\hline 0315-0057897 & $11.51 \pm 1.42$ & 0.52 & $-10.0 \pm 1.3$ & $-19.7 \pm 1.0$ & $-2.7 \pm 0.7$ & 2.69 & 3.73 & $\mathrm{~N}$ & CAR \\
\hline $0352-0070710$ & $8.50 \pm 0.67$ & -0.04 & $-22.2 \pm 2.0$ & $-17.9 \pm 0.5$ & $-5.2 \pm 1.0$ & 2.10 & 3.47 & $\mathrm{~N}$ & ARG \\
\hline 0263-0084896 & $10.69 \pm 1.04$ & 0.36 & $-10.1 \pm 1.3$ & $-23.1 \pm 0.4$ & $-4.1 \pm 0.5$ & 0.01 & 0.29 & $\mathrm{~N}$ & CAR \\
\hline $0331-0114466$ & $9.69 \pm 1.32$ & 0.31 & $-10.0 \pm 1.6$ & $-23.6 \pm 0.3$ & $-2.9 \pm 0.8$ & 0.42 & 1.65 & $\mathrm{~N}$ & $\mathrm{CAR}$ \\
\hline 0406-0105889 & $8.19 \pm 1.02$ & 0.29 & $-13.6 \pm 1.7$ & $-22.8 \pm 0.4$ & $-4.4 \pm 1.1$ & 0.71 & 1.82 & $\mathrm{~N}$ & $\mathrm{COL}$ \\
\hline 0248-0110675 & $9.98 \pm 1.04$ & 0.27 & $-10.2 \pm 1.6$ & $-23.1 \pm 0.5$ & $-4.8 \pm 0.7$ & 0.03 & 0.44 & $\mathrm{~N}$ & CAR \\
\hline 0354-0106699 & $8.37 \pm 0.67$ & 0.41 & $-22.0 \pm 2.2$ & $-14.8 \pm 0.4$ & $-4.7 \pm 1.4$ & 0.03 & 0.50 & $\mathrm{~N}$ & ARG \\
\hline 0341-0108561 & $9.44 \pm 0.99$ & 0.33 & $-21.6 \pm 2.9$ & $-14.1 \pm 0.8$ & $-7.4 \pm 2.1$ & 0.31 & 2.41 & $\mathrm{~N}$ & $\mathrm{ARG}$ \\
\hline 0489-0152365 & $8.39 \pm 0.97$ & 0.26 & $-13.1 \pm 1.3$ & $-24.2 \pm 0.5$ & $-6.6 \pm 1.1$ & 2.40 & 2.54 & $\mathrm{~N}$ & $\mathrm{COL}$ \\
\hline 0269-0122275 & $8.09 \pm 1.07$ & -0.02 & $-10.1 \pm 2.3$ & $-23.5 \pm 0.8$ & $-5.1 \pm 1.6$ & 0.10 & 0.84 & $\mathrm{~N}$ & CAR \\
\hline 0291-0099052 & $9.20 \pm 1.21$ & 0.49 & $-10.0 \pm 2.2$ & $-24.0 \pm 0.6$ & $-2.0 \pm 1.5$ & 0.79 & 2.62 & $\mathrm{~N}$ & CAR \\
\hline $0372-0169783$ & $12.14 \pm 1.49$ & 0.94 & $-9.7 \pm 1.7$ & $-23.3 \pm 0.5$ & $-5.9 \pm 1.2$ & 0.27 & 1.63 & $\mathrm{~N}$ & CAR \\
\hline 0313-0102916 & $8.52 \pm 1.01$ & 0.29 & $-10.0 \pm 1.9$ & $-22.7 \pm 0.7$ & $-5.6 \pm 1.1$ & 0.16 & 1.25 & $\mathrm{~N}$ & CAR \\
\hline 0397-0143387 & $10.28 \pm 2.08$ & 0.92 & $-10.7 \pm 3.1$ & $-22.7 \pm 0.1$ & $-2.5 \pm 2.1$ & 0.23 & 1.95 & $\mathrm{~N}$ & CAR \\
\hline 0308-0116109 & $8.63 \pm 1.08$ & 0.57 & $-10.2 \pm 2.1$ & $-23.0 \pm 1.1$ & $-4.1 \pm 1.3$ & 0.01 & 0.30 & $\mathrm{Y}$ & $\mathrm{CAR}^{*}$ \\
\hline 0415-0155444 & $10.55 \pm 0.97$ & 0.18 & $-10.5 \pm 1.1$ & $-21.9 \pm 0.4$ & $-3.6 \pm 0.6$ & 0.60 & 1.38 & $\mathrm{~N}$ & CAR \\
\hline $0193-0211246$ & $9.34 \pm 0.93$ & 0.09 & $-10.1 \pm 1.9$ & $-23.2 \pm 0.9$ & $-4.1 \pm 1.2$ & 0.02 & 0.35 & $\mathrm{~N}$ & CAR \\
\hline 0367-0179130 & $8.34 \pm 1.35$ & 0.03 & $-10.1 \pm 2.7$ & $-23.6 \pm 0.8$ & $-4.6 \pm 1.9$ & 0.10 & 0.68 & $\mathrm{~N}$ & CAR \\
\hline $0327-0202270$ & $8.67 \pm 0.89$ & -0.02 & $-10.4 \pm 1.8$ & $-22.2 \pm 2.3$ & $-4.1 \pm 1.2$ & 0.05 & 0.91 & $\mathrm{Y}$ & $\mathrm{CAR}^{*}$ \\
\hline $0154-0097276$ & $12.26 \pm 0.89$ & 0.26 & $-10.1 \pm 1.4$ & $-23.1 \pm 0.7$ & $-5.0 \pm 0.6$ & 0.05 & 0.63 & $\mathrm{~N}$ & CAR \\
\hline $0370-0341486$ & $21.19 \pm 1.30$ & -0.02 & $-22.2 \pm 1.7$ & $-14.1 \pm 1.2$ & $-4.6 \pm 0.6$ & 0.04 & 0.53 & $\mathrm{~N}$ & ARG \\
\hline 0104-0056948 & $13.22 \pm 0.99$ & 0.65 & $-6.9 \pm 1.6$ & $-28.6 \pm 1.0$ & $-10.5 \pm 0.6$ & 1.13 & 3.08 & $\mathrm{~N}$ & $\mathrm{ABD}$ \\
\hline $0277-1048331$ & $18.57 \pm 0.82$ & 0.15 & $-11.0 \pm 5.8$ & $-15.6 \pm 3.0$ & $-8.5 \pm 2.8$ & 0.03 & 1.14 & $\mathrm{Y}$ & BPMG \\
\hline 0601-0968580 & $12.83 \pm 1.26$ & 0.08 & $-7.9 \pm 0.5$ & $-15.9 \pm 2.2$ & $-9.5 \pm 1.9$ & 0.34 & 2.19 & $\mathrm{Y}$ & BPMG \\
\hline $0438-0823866$ & $14.32 \pm 0.83$ & 0.04 & $-7.3 \pm 1.2$ & $-16.4 \pm 1.3$ & $-10.3 \pm 1.1$ & 0.67 & 3.06 & $\mathrm{~N}$ & BPMG \\
\hline $0577-1232093$ & $19.21 \pm 1.19$ & -0.09 & $-9.3 \pm 1.2$ & $-15.0 \pm 1.2$ & $-10.5 \pm 1.2$ & 0.36 & 1.71 & $\mathrm{~N}$ & BPMG \\
\hline
\end{tabular}

U,V,W values for 0540-0027893 using the measured trigonometric parallax of HD 21423 instead of the kinematic one, we found that the new values of $\mathrm{U}=-13.7 \pm 0.8, \mathrm{~V}=$ $-22.7 \pm 1.0, \mathrm{~W}=-4.7 \pm 0.9 \mathrm{~km} \mathrm{~s}^{-1}$ are also very similar to the UVW values of COL (the recomputed $\chi_{r}^{2}$ and $d_{U V W}$ values are 0.42 and $1.6 \mathrm{~km} \mathrm{~s}^{-1}$ ). Taking all these into account, it is likely that HD 21423, HD 21434, and 0540-0027893 constitute a widely separated multiple system and they are secure members of COL. HD 21423 has a spectral type of A1V, while HD 21434 is an A9V-type star, according to SIMBAD. HD 21434 itself is a close binary with a separation of 0.2 (Mason et al. 2001). At a parallax of 9.27 mas $\mathrm{yr}^{-1}$, the minimum separation of 0540-0027893 to HD 21423 and HD 21434 would be $\sim 9170$ AU and $\sim 11540$ AU, respectively.

0454-0034540 (CD--44 1533): This star was observed in the framework of the SACY survey (Torres et al. 2006) and identified as a K0 dwarf with a $v \sin i$ of $34 \pm 0.5 \mathrm{~km} \mathrm{~s}^{-1}$. We classified it as a probable member of COL based on its measured properties. The high $v \sin i$ value also supports the youth of this target. Its radial velocity, measured in the $R A V E$ survey (Siebert et al. 2011), $v \sin i=$
$19.8 \pm 2.0 \mathrm{~km} \mathrm{~s}^{-1}$ is well consistent with the SACY-based RV value of $+19.3 \pm 0.2 \mathrm{~km} \mathrm{~s}^{-1}$.

0466-0044706 and 0466-0044732 (HIP 25434 and HIP 25436): We identified these stars as secure members of COL. 0466-0044706 was classified as a G0-type star, while 0466-0044732 has a spectral type of F4IV/V. According to Mason et al. (2001), 0466-0044732 (component A) and 0466-0044706 (B) constitute a visual binary system with a separation of $12^{\prime \prime}$. Based on the NOMAD catalogue the

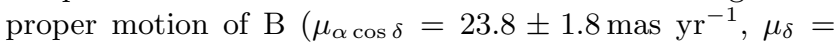
$\left.12.4 \pm 1.8 \mathrm{mas} \mathrm{yr}^{-1}\right)$ is consistent with that of $\mathrm{A}\left(\mu_{\alpha} \cos \delta=\right.$ $\left.21.4 \pm 2.3 \mathrm{mas}, \mu_{\delta}=13.2 \pm 2.7 \mathrm{mas}\right)$. Their Hipparcos paral-

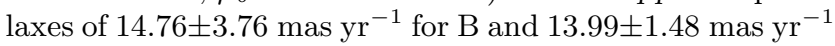
for A agree well within the quoted uncertainties. Our RV values of $23.6 \pm 1.8 \mathrm{~km} \mathrm{~s}^{-1}$ and $22.6 \pm 6.7 \mathrm{~km} \mathrm{~s}^{-1}$ for B and A, respectively, seems to be consistent as well.

Desidera et al. (2006) also obtained highresolution spectra for these stars and reported RVs of $24.94 \pm 0.33 \mathrm{~km} \mathrm{~s}^{-1}$ and $32.17 \pm 1.3 \mathrm{~km} \mathrm{~s}^{-1}$ for the B and A components, respectively. As an explanation for the observed RV difference, they suggested that some of the components might host a further companion. According 
to this work, the G0-type B component shows strong chromospheric activity $\left(\log R_{H K}^{\prime}=-4.3\right)$. This large $R_{H K}^{\prime}$ value, together with the measured high projected rotational velocities ( 110 and $40 \mathrm{~km} \mathrm{~s}^{-1}$ for the A and B components, respectively) also supports the young age of these stars.

0211-0114754 (HD 269620): This G6V-type star was also assigned to COL. The lithium equivalent width and the radial velocity of the star were taken from the SACY catalogue (Torres et al. 2006) that also provided a $v \sin i$ value of $22.3 \pm 3.1 \mathrm{~km} \mathrm{~s}^{-1}$ for this object.

0211-0129478 (HD 269921): This G7V-type star is located relatively close to 0211-0114754 in the sky (separation $\left.\sim 49^{\prime}\right)$. It was also observed in the framework of the SACY survey. This star is a fast rotator $\left(v \sin i=70 \mathrm{~km} \mathrm{~s}^{-1}\right.$, Torres et al. 2006). It was assigned to COL as a probable member in our analysis.

0397-0143387 (TYC 8174-1586-1): The proper motions $\left(\mu_{\alpha \cos \delta}=-185.6 \pm 1.3 \operatorname{mas~yr}^{-1}, \mu_{\delta}=-99.2 \pm 2.5 \mathrm{mas} \mathrm{yr}^{-1}\right.$ ) included in the TYCHO2 catalogue (Høg et al. 2000) and proper motions $\left(\mu_{\alpha \cos \delta}=-19.8 \pm 4.5\right.$ mas $\mathrm{yr}^{-1}, \mu_{\delta}=$

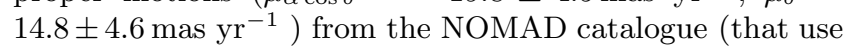
the UCAC2 catalogue data Zacharias et al. 2004b, for this object) significantly differ, raising the question which astrometric data is correct. The NOMAD data are consistent with the proper motion data of the UCAC4 $\left(\mu_{\alpha \cos \delta}=-15.9 \pm\right.$

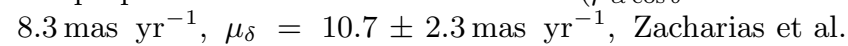
$2013)$ and of the SPM4 $\left(\mu_{\alpha \cos \delta}=-19.45 \pm 2.6\right.$ mas $\mathrm{yr}^{-1}$, $\mu_{\delta}=7.42 \pm 2.61$ mas $\mathrm{yr}^{-1}$, Girard et al. 2011) catalogues. However, we note that the latter catalogues are somewhat dependent on each other e.g. SPM4 and UCAC4 share some first epoch data (Zacharias et al. 2013). According to Torres et al. (2006), 0397-0143387 is a K5eV type star. Assuming that it is a normal main-sequence star, its absolute V-band magnitude is $M_{V} \sim 7.35$ based on Drilling \& Landolt (2000). Using this absolute magnitude to derive a photometric distance for the object, and adopting the TYCHO astrometric data and the RV taken from Torres et al. (2006), we computed the U, V, and W galactic space velocities of $-21.0,-21.0,-80.7 \mathrm{kms}^{-1}$, respectively. Both the high lithium content $\left(\mathrm{EW}_{\mathrm{Li}}=340 \mathrm{~m} \AA\right)$ and large X-ray fractional luminosity $\left(\log L_{x} / L_{b o l}=-3.18\right)$ of 0397-0143387 indicate that this star is probably younger than 100 Myr. According to Leggett (1992), young disk population stars occupy a well defined region in the 3dimensional $\mathrm{U}, \mathrm{V}, \mathrm{W}$ space. The $\mathrm{U}, \mathrm{V}, \mathrm{W}$ velocities obtained from TYCHO proper motion data are clearly outside this box. The obtained large velocity dispersion would rather imply a quite old star. Therefore, we consider the NOMAD/UCAC4/SPM4 data more reliable in this case, i.e. this star has a space velocity consistent with that of the Carina group.

0277-1048331 (HD 173167): This F5V-type star was found to be a probable member of BPMG. It is located close to TYC 9073-0762 (with a separation of $\sim 550^{\prime \prime}$ ) that was proposed by Torres et al. (2006) to be a member of BPMG. These two stars have very similar proper motions, $\mu_{\alpha \cos \delta}=16.1 \pm 1.3$ mas $\mathrm{yr}^{-1}, \mu_{\delta}=-80.2 \pm 1.3{\mathrm{mas} \mathrm{yr}^{-1}}^{-1}$

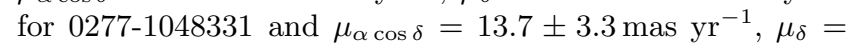

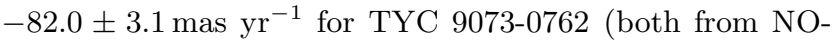
MAD), suggesting that they could be companions forming a wide separation binary. We found that 0277-1048331 may be a spectroscopic binary with a measured systemic radial velocity of $+0.8 \pm 7.0 \mathrm{~km} \mathrm{~s}^{-1}$ which is in agreement with the $\mathrm{RV}$ of $+2.4 \pm 0.1 \mathrm{~km} \mathrm{~s}^{-1}$ measured at TYC 9073-0762 in the SACY survey, however, the uncertainty of the former value is quite high. The estimated kinematic distance of this system is $54 \mathrm{pc}$ both based on our analysis and Torres et al. (2006). According to Torres et al. (2006), TYC 9073-0762 is an M1Ve type star with a very high $\mathrm{EW}_{\mathrm{Li}}$ of $332 \mathrm{~mA}$.

0601-0968580: Although lithium was not detected in its spectrum, this star was classified as a probable member of BPMG because it may form a binary system with TYC 6872-1011-1, a previously identified member of BPMG (Torres et al. 2006). They measured an RV and $v \sin i$ of $+4.9 \pm 1.0 \mathrm{~km} \mathrm{~s}^{-1}$ and $33.8 \pm 3.4 \mathrm{~km} \mathrm{~s}^{-1}$, respectively, and identified lithium absorption in the spectrum with an equivalent width of $483 \mathrm{~mA}$. The separation between 06010968580 and TYC $6872-1011-1$ is only $28^{\prime \prime} .3$ and both the measured radial velocities $\left(-7.0 \pm 0.3 \mathrm{~km} \mathrm{~s}^{-1}\right.$ for 06010968580) and the proper motion vectors $\left(\mu_{\alpha \cos \delta}=12.9 \pm\right.$

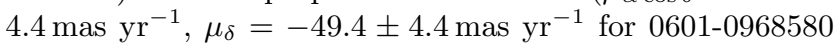

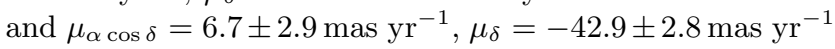
for TYC 6872-1011-1) are quite consistent with each other, suggesting that the two stars constitute a physical binary system.

0438-0823866 (2MASS J19233820-4606316): We assigned this M0-type star to BPMG as a probable new member. It has an $\mathrm{EW}_{\mathrm{Li}}=422 \pm 12 \mathrm{m \AA}$ (this work) confirming that its age is similar to the kinematic group assigned. Malo et al. (2013) also found it to be a probable candidate of BPMG. Their measured radial velocity of $-0.7 \pm 0.7 \mathrm{~km} \mathrm{~s}^{-1}$ is in good agreement with our radial velocity value of $-0.2 \pm 1.1 \mathrm{~km} \mathrm{~s}^{-1}$.

0577-1232093 (2MASS J20055640-3216591): Our analysis identified $0577-1232093$ as a probable new member of BPMG. Lithium absorption line was clearly detected in its spectrum with an $\mathrm{EW}_{\mathrm{Li}}=140 \pm 16 \mathrm{~m} \AA$ that is quite consistent with the lithium equivalent widths measured in known BPMG members with similar colour indices (Fig. 3).

\subsubsection{Other targets (rejected candidates)}

0298-0039269 (HD 37402): Although this F6V-type star may be as old as the Columba group according to its lithium content and strong X-ray activity, we do not classify it as a member because it does not entirely fulfill our kinematic criteria. Its $\chi_{r}^{2}$ is 5.2, lower than the threshold value of 6 , but its $d_{U V W}=5.3 \mathrm{~km} \mathrm{~s}^{-1}$ is somewhat larger than the threshold value of $d_{U V W}=5.0 \mathrm{~km} \mathrm{~s}^{-1}$. Additional, more accurate observations of radial velocity and astrometric parameters are needed to better assess its membership. We note that the star is included in the Hipparcos catalogue.

0348-0138354 (TYC 8587-1015-1), 0393-0168117: We detected lithium absorption line in the spectra of both targets. On the one hand the large measured equivalent widths of $252 \pm 14 \mathrm{m \AA}$ for 0348-0138354 and of 
$277 \pm 10 \mathrm{m \AA}$ for 0393-0168117 with their high measured X-ray fractional luminosities (Table 3) suggest an age of $<50$ Myr for these targets. On the other hand, their Galactic space motion does not seem to match the space motion of known young nearby moving groups. However, 0348-0138354 was found to be a spectroscopic binary in our analysis implying that its radial velocity measurement is less reliable. Further spectroscopic observations are needed to clarify whether the rejection of this candidate was correct or not.

0405-0785226 (2MASS J18151564-4927472): Although its kinematic properties match those of BPMG, we do not list it as a probable member due to the lack of detectable amount of lithium in the spectrum. We cannot state that it is very young $(\sim 10-20 \mathrm{Myr})$ based solely on its high fractional X-ray luminosity. We note that Malo et al. (2013) also identified it as a candidate member of BPMG (see their table 5).

0706-0872415 (2MASS J21100535-1919573): Both the galactic space motion and space position of the source are found to be consistent with the respective properties of BPMG, however, based on our current age diagnostic, the co-evality of the system with BPMG is not satisfactorily established. Therefore it has not been assigned as a probable member of BPMG yet. This star was also classified as a candidate member of BPMG in Malo et al. (2013).

\section{SUMMARY}

With the aim to explore the stellar content of five nearby young moving groups ( $\beta$ Pic, Tucana-Horologium, Columba, Carina, Argus) further, we searched for new members in these kinematic assemblages, by combining astrometric, photometric, X-ray, and high-resolution spectroscopic data. Initially, we used the proper motion based algorithm proposed by Lépine \& Simon (2009) to identify those stars from a cross-correlated NOMAD/ROSAT catalogues that have proper motions consistent with membership of any of these groups. This list was further refined by selecting only those targets whose fractional X-ray luminosity and position in the colour-magnitude diagram were consistent with those of the known members. As a result of this selection algorithm, we finally identified more than 100 stars that could be potentially assigned to at least one of the selected five young moving groups.

In order to further assess their membership status, we obtained high-resolution spectra for fifty candidate stars using the Echelle spectrograph at the 2.3-m telescope of the Australian National University and the FEROS instrument at the $2.2 \mathrm{~m} \mathrm{MPG/ESO} \mathrm{telescope.} \mathrm{For} \mathrm{four} \mathrm{additional} \mathrm{ob-}$ jects, we used data from the SACY survey (Torres et al. 2006) to complement our observed sample. Radial velocity data and lithium equivalent widths extracted from these spectra offered us the opportunity to perform a more accurate kinematic and age analysis of our candidates and to select targets with kinematic properties and ages consistent with those of the specific groups.

Based on this analysis, we identified 33 new probable young members of BPMG (4 stars), COL (11 stars), CAR (14 stars), and ARG (4 stars) kinematic groups, as well as two new possible members of CAR. In addition we found serendipitiously a new AB Dor moving group member. Trigonometric parallax measurements are needed to securely confirm the membership status of these stars. For four COL stars (HD 3888, HD 8077, HIP 25434, HIP 25436) Hipparcos based parallaxes have already been available, thus they can be considered as secure new members. Moreover, we found that 0540-0027893, which is also assigned to COL, may be located in a wide multiple system. HD 21423, the most massive companion in this multiple system, has a Hipparcos parallax, which is perfectly consistent with the predicted kinematic parallax of 0540-0027893, making the membership of this system in Columba to be secure. We note that the membership of HD 21423 and HD 21434, the two early-type (A1V and $\mathrm{A} 9 \mathrm{~V}$ ) companions is also a new discovery. Two new probable BPMG members, 0277-1048331 and 0601-0968580 seem to form binary systems with two previously identified members of this group (TYC 9073-0762 and TYC 68721011-1, respectively). We significantly increased the number of known probable/possible members in the Carina group by adding 16 new stars to those 23 already listed in Torres et al. (2008).

Our preselection method turned out to be very successful. From the 54 candidates, 34 were verified as probable young moving group members based on the analysis of highresolution spectroscopic data.

\section{ACKNOWLEDGEMENTS}

We thank our anonymous referee whose comments significantly improved the manuscript. This project was supported by the Hungarian OTKA grants K76816, K83790, K101393, K104607, the HUMAN MB08C 81013 project of the MAG Zrt., the PECS-98073 program of the European Space Agency (ESA) and the Hungarian Space Office, the Lendület 2009/2012 Young Researchers' Programs and the Bolyai Research Fellowship of the Hungarian Academy of Sciences. This publication makes use of data products from the Wide-field Infrared Survey Explorer, which is a joint project of the University of California, Los Angeles, and the Jet Propulsion Laboratory/California Institute of Technology, funded by the National Aeronautics and Space Administration. The publication also makes use of data products from the Two Micron All Sky Survey, which is a joint project of the University of Massachusetts and the Infrared Processing and Analysis Center/California Institute of Technology, funded by the National Aeronautics and Space Administration and the National Science Foundation. Our research has made use of the VizieR catalogue access tool, CDS, Strasbourg, France.

\section{REFERENCES}

Barrado y Navascués D., Stauffer J. R., Song I., Caillault J.-P., 1999, ApJ, 520, L123

Barrado y Navascués D., Martín E. L., 2003, AJ, 126, 2997

Barrado y Navascués D., Stauffer J. R., Jayawardhana R., 2004, ApJ, 614, 386

Carson J., et al., 2013, ApJ, 763, L32

Chambers J. E., 2001, Icar, 152, 205 
da Silva L., et al., 2009, A\&A, 508, 833

Desidera S., Gratton R. G., Lucatello S., Claudi R. U., Dall T. H., 2006, A\&A, 454, 553

Drilling J. S. \& Landolt A. U., Infrared Astronomy, in Cox A. N., ed., Allen's Astrophysical Quantities, 4th edn. AIP Press, New York, ISBN: 0387987460. 2000. p. 388.

Fernández D., Figueras F., Torra J., 2008, A\&A, 480, 735

Girard T. M., et al., 2011, AJ, 142, 15

Grenier S., Burnage R., Faraggiana R., Gerbaldi M., Delmas F., Gómez A. E., Sabas V., Sharif L., 1999, A\&AS, 135,503

Hauschildt P. H., Allard F., Baron E., 1999, ApJ, 512, 377

Høg E., et al., 2000, A\&A, 355, L27

Jayawardhana R., Coffey J., Scholz A., Brandeker A., van Kerkwijk M. H., 2006, ApJ, 648, 1206

Kasper M., et al., 2007, A\&A, 472, 321

Kaufer A., Stahl O., Tubbesing S., Nørregaard P., Avila G., Francois P., Pasquini L., Pizzella A., 1999, Msngr, 95, 8

Kenyon S. J., Bromley B. C., 2006, AJ, 131, 1837

Kenyon S. J., Bromley B. C., 2008, ApJS, 179, 451

Kiss L. L., et al., 2011, MNRAS, 411, 117

Lagrange A.-M., et al., 2010, Sci, 329, 57

Lallement R., Welsh B. Y., Vergely J. L., Crifo F., Sfeir D., 2003, A\&A, 411, 447

Leggett S. K., 1992, ApJS, 82, 351

Lépine S., Simon M., 2009, AJ, 137, 3632

Makarov V. V., 2007, ApJS, 169, 105

Malo L., Doyon R., Lafrenière D., Artigau É., Gagné J., Baron F., Riedel A., 2013, ApJ, 762, 88

Marois C., Macintosh B., Barman T., Zuckerman B., Song I., Patience J., Lafrenière D., Doyon R., 2008, Sci, 322, 1348

Mason B. D., Wycoff G. L., Hartkopf W. I., Douglass G. G., Worley C. E., 2001, AJ, 122, 3466

Mentuch E., Brandeker A., van Kerkwijk M. H., Jayawardhana R., Hauschildt P. H., 2008, ApJ, 689, 1127

Moór A., et al., 2011, ApJS, 193, 4

Munari U., Sordo R., Castelli F., Zwitter T., 2005, A\&A, 442,1127

Ortega V. G., de la Reza R., Jilinski E., Bazzanella B., 2002, ApJ, 575, L75

Pascucci I., et al., 2006, ApJ, 651, 1177

Pecaut M. J., Mamajek E. E., 2013, ApJ, accepted, arXiv:1307.2657

Rice E. L., Faherty J. K., Cruz K. L., 2010, ApJ, 715, L165

Riedel A. R., Murphy S. J., Henry T. J., Melis C., Jao

W.-C., Subasavage J. P., 2011, AJ, 142, 104

Schlieder J. E., Lépine S., Simon M., 2010, AJ, 140, 119

Schlieder J. E., Lépine S., Simon M., 2012, AJ, 143, 80

Schlieder J. E., Lépine S., Simon M., 2012, AJ, 144, 109

Shkolnik E., Liu M. C., Reid I. N., 2009, ApJ, 699, 649

Shkolnik E. L., Liu M. C., Reid I. N., Dupuy T., Weinberger A. J., 2011, ApJ, 727, 6

Shkolnik E. L., Anglada-Escudé G., Liu M. C., Bowler B. P., Weinberger A. J., Boss A. P., Reid I. N., Tamura M., 2012, ApJ, 758, 56

Siebert A., et al., 2011, AJ, 141, 187

Skrutskie M. F., et al., 2006, AJ, 131, 1163

Song I., Zuckerman B., Bessell M. S., 2003, ApJ, 599, 342

Torres C. A. O., da Silva L., Quast G. R., de la Reza R., Jilinski E., 2000, AJ, 120, 1410
Torres C. A. O., Quast G. R., de La Reza R., da Silva L., Melo C. H. F., Sterzik M., 2003, ASSL, 299, 83

Torres C. A. O., et al., 2006, A\&A, 460, 695

Torres C. A. O., et al., 2008, Handbook of Star Forming Regions, Vol. II, ASP Monograph Publications, Vol. 5, 757

Zacharias N., Monet D. G., Levine S. E., Urban S. E., Gaume R., Wycoff G. L., 2004, AAS, 36, 1418

Zacharias N., Urban S. E., Zacharias M. I., Wycoff G. L., Hall D. M., Monet D. G., Rafferty T. J., 2004, AJ, 127, 3043

Zacharias N., Finch C. T., Girard T. M., Henden A., Bartlett J. L., Monet D. G., Zacharias M. I., 2013, AJ, 145,44

Zuckerman B., Webb R. A., 2000, ApJ, 535, 959

Zuckerman B., Song I., Bessell M. S., Webb R. A., 2001, ApJ, 562, L87

Zuckerman B., Song I., Webb R. A., 2001, ApJ, 559, 388

Zuckerman B., Song I., 2004, ARA\&A, 42, 685

Zuckerman B., Rhee J. H., Song I., Bessell M. S., 2011, ApJ, 732, 61

Zuckerman B., Song I., 2012, ApJ, 758, 77

van Leeuwen, F. 2007, Astrophysics and Space Science Library, 350, by F. van Leeuwen

Voges W., et al., 1999, A\&A, 349, 389

Voges W., et al., 2000, IAUC, 7432, 1

White R. J., Basri G., 2003, ApJ, 582, 1109

Wright E. L., et al., 2010, AJ, 140, 1868

Wyatt M. C., 2008, ARA\&A, 46, 339

\section{APPENDIX A: DESCRIPTION OF THE SELECTED FIVE YOUNG ASSOCIATIONS}

BPMG was first proposed by Zuckerman et al. (2001). After Barrado y Navascués et al. (1999) found two young Mtype proper motion companions to $\beta$ Pic, Zuckerman et al. (2001) defined this group by identifying 18 systems that likely belong to it. In their review work, Torres et al. (2008) listed 48 high probability members, most of them concentrated in the southern hemisphere, with distances ranging between 9 and 80 pc. Thanks to recent studies (Lépine \& Simon 2009; Rice, Fahertv, \& Cruz 2010; Schlieder. Lépine, \& Simon 2010; Kiss et al. 2011; Moór et al. 2011; Schlieder, Lépine, \& Simon 2012b; Malo et al. 2013), the number of probable members has been increased considerably in the last few years, with many newly identified systems located in the northern hemisphere (e.g. Schlieder, Lépine, \& Simon 2010, 2012b; Moór et al. 2011). Based on the members' location in the H-R diagram and from their lithium equivalent widths, Zuckerman et al. (2001) derived an age of $12_{-4}^{+8} \mathrm{Myr}$ for the group. Using dynamical back-tracing models of members, Ortega et al. (2002) and Song. Zuckerman. \& Bessell (2003) obtained very similar age estimates of $12 \mathrm{Myr}$ for this group. Recently Mentuch et al. (2008) derived a somewhat higher age, $21 \pm 9 \mathrm{Myr}$, from lithium depletion in the group.

Tucana-Horologium, Columba and Carina moving groups together constitute the so-called Great Austral Young Association (GAYA, Torres et al. 2008). Considering Li abundances, these three groups are indistinguishable, 


\section{4}

$$
\text { A. Moór et al. }
$$

indicating almost identical ages (da Silva et al. 2009). Members of these groups can be separated based on their galactic space motion and galactic space location, although the characteristic $\mathrm{U}, \mathrm{V}, \mathrm{W}$ space velocities of COL are quite similar to those of CAR and the space regions occupied by their members also overlap partially making the accurate group assignment difficult in some cases. THA is the nearest with a mean distance of $\sim 48 \mathrm{pc}$, and therefore it was discovered first (Zuckerman \& Webb 2000; Torres et al. 2000) among the three groups. COL and CAR groups were proposed later as separate entities by Torres et al. (2008). Prior to that, some COL and CAR members were assigned to the GAYA2 group (Torres et al. 2003). Torres et al. (2008) quoted 44, 53, and 23 probable members of THA, COL, and CAR, respetively. da Silva et al. (2009) added six new stars to COL, but also rejected three from those listed in Torres et al. (2008). Recently Zuckerman et al. (2011) and Zuckerman \& Song (2012) contributed significantly to the census of THA and COL by proposing in total 12 and 17 new members, respectively. The majority of these stars are early-type with spectral types A or F. Kiss et al. (2011) and Moór et al. (2011) also identified some additional probable THA and COL members. Similarly to BPMG, most members of the GAYA complex are located in South. The age estimates for THA range between 10 and $40 \mathrm{Myr}$ (Zuckerman, Song. \& Webb 2001; Makarov 2007; Mentuch et al. 2008; da Silva et al. 2009). The most widely accepted value at the moment is 30 Myr, which is the proposed age of the whole GAYA complex (Torres et al. 2008).

Argus association. The existence of the Argus association was proposed by Torres et al. (2003). ARG comprises both a sparsely distributed "classic" moving group and members of the IC 2391 open cluster (Torres et al. 2008). The 29 members identified in the sparse part of the group are usually found to be closer to us than stars that belong to IC 2391 that are all located at a distance $>120$ pc. Zuckerman et al. (2011) and Zuckerman \& Song (2012) found 13 new nearby ( $<80$ pc) early-type Argus members. According to Riedel et al. (2011), the nearest pre-main sequence star, AP Col, with a distance of 8.4 pc can also be assigned to this group. Based on the members' position in the H-R diagram and on their lithium equivalent widths, Torres et al. (2008) derived an age of 40 Myr for ARG. Barrado y Navascués, Stauffer, \& Javawardhana (2004) obtained an age estimate of $50 \pm 5$ Myr for IC 2391 based on the position of the "Li depletion boundary". Comparing the position of recently proposed A-type members of Argus with the location of Pleiades stars, Zuckerman \& Song (2012) suggested that the group may be somewhat older than the previously determined $40 \mathrm{Myr}$. The Argus association is also concentrated in the Southern hemisphere. 